Running head: Memory \& Mind-Wandering

\title{
Targeted Memory Reactivation during Mind-Wandering in Younger and Older Adults
} Jessica Nicosia \& David A. Balota

Washington University in St. Louis

\section{UNPUBLISHED MANUSCRIPT UNDER PEER REVIEW}

\author{
Author Note \\ Jessica Nicosia, Department of Psychological and Brain Sciences, Washington University in St. \\ Louis; David A. Balota, Department of Psychological and Brain Sciences, Washington \\ University in St. Louis. Aspects of this article were presented to the Graduate School of Arts and \\ Sciences at Washington University in St. Louis as a dissertation by Jessica Nicosia. Aspects of \\ this article were also presented in a poster at the 2021 Annual Meeting of the Psychonomic \\ Society. The research was supported by an NIA Training Grant T32-AG000030-44. \\ Correspondence should be addressed to j.nicosia@wustl.edu. All data, code, and study materials \\ are available upon request.
}




\begin{abstract}
Mind-wandering (MW) is a universal cognitive process that is estimated to comprise $\sim 30 \%$ of our everyday thoughts. Despite its prevalence, the functional utility of MW remains a scientific blind spot. The present study sought to investigate whether MW serves a functional role in cognition. Specifically, we investigated whether MW contributes to memory consolidation processes, and if age differences in the ability to reactivate episodic memories during MW may contribute to age-related declines in episodic memory. Younger and older adults encoded paired associates, received targeted reactivation cues during an interval filled with a task which promotes MW, and were tested on their memory for the cued and uncued stimuli from the initial encoding task. Thought probes were presented during the retention (MW) interval to assess participants' thought contents. Across three experiments, we compared the effect of different cue modalities (i.e., auditory, visual) on cued recall performance, and examined both correct retrieval response times as well as accuracy. Across experiments, there was evidence that stimuli that were cued during the MW task were correctly retrieved more quickly than uncued stimuli and that this effect was more robust for younger adults than older adults. Additionally, the more MW a participant reported during the retention interval, the stronger the cueing effect they produced during retrieval. The results from these experiments are interpreted within a retrieval facilitation framework wherein cues serve to reactivate the earlier traces during MW, and this reactivation benefits retrieval speed for cued items as compared to uncued items.
\end{abstract}

Keywords: episodic memory, consolidation, mind-wandering, aging Abstract Word Count: 247/250 | Body Word Count: 10,628 


\section{Targeted Memory Reactivation during Mind-Wandering in Younger and Older Adults}

Mind-wandering (MW) was originally conceptualized as a central element of our "stream of consciousness" (James, 1892). The generic term MW includes many behaviors such as “daydreaming” (Giambra, 1979), “task-unrelated images and thought” (Giambra and Grodsky, 1989), "stimulus independent thought" (Teasdale et al., 1995), "incidental self-processing” (Gilbert et al., 2005), “inner speech" (Morin, 2009), and "spontaneous thought” (Christoff et al., 2011). The topic has garnered considerable attention from the fields of philosophy, psychology, and neuroscience and has rapidly gained interest in the past two decades (Callard et al., 2013). One reason that MW research has gained traction is due to its ubiquity across individuals and in our everyday lives. For example, it is estimated that about $30 \%$ of our everyday thoughts consist of MW (Kane et al., 2007; Klinger \& Cox, 1987) and 96\% of American adults report experiencing daydreaming every day (Singer \& McCraven, 1961).

Although there is considerable interest in MW, the nature of how MW interacts with other cognitive processes remains a "scientific blind spot" (Gruberger et al., 2011). Some researchers have suggested that MW may simply be a default mental state which does little to contribute to other cognitive functions (Wang et al., 2009). In contrast, researchers have recently suggested that it may serve a more functional role (see Mildner \& Tamir, 2019). Specifically, given that the contents of MW include everything from the direct recapitulation of recent experiences to variations on past events and imagined future scenarios (Andrews-Hannah et al., 2013; Klinger \& Cox, 1987; Smallwood \& Schooler, 2015; Wamsley, 2019), it has recently been proposed that such offline memory replay during MW may serve an important role in processes related to memory consolidation (Mildner \& Tamir, 2019). Memory Consolidation, Wakeful Rest, and Mind-Wandering 
Memory consolidation can be defined as a process where memories gradually become independent of the hippocampus as they are transferred to diffuse neocortical regions for later retrieval (Wixted \& Cai, 2014). Although the potential mechanisms through which the hippocampus and neocortex communicate are not well understood, one leading candidate is neural replay (Klinzing et al., 2019). Initially observed in the hippocampal cells of rodents, the phenomenon of hippocampal replay reflects the fact that the hippocampal place cells that fired during encoding tended to sequentially re-activate during post-encoding sleep (O'Keefe \& Distrovsky, 1971; Wilson \& McNaughton, 1994). It is hypothesized that this replay of earlier experiences may be necessary for reorganizing representations in the neocortex and thus critical for consolidation. Several studies have investigated neural replay in humans as well. For example, Rasch et al. (2007) showed that cueing recently formed odor-associated memories via odor re-exposure during sleep prompted hippocampal re-activation (as measured by fMRI) and resulted in less forgetting after sleep as compared to uncued and awake control groups. In addition, Peigneugx et al. (2004) showed that the degree of overlapping reactivation (again, as measured by fMRI) during post-learning sleep is associated with post-sleep memory performance.

Although most work on hippocampal replay has examined the phenomenon during sleep, recent studies have investigated the possibility that some offline, awake states may also promote consolidation. Importantly, however, the majority of studies which have shown the benefit of sleep on memory retention have compared sleep to active awake conditions (i.e., participants watch videos, listen to music, or go about their daily activities; Lau et al., 2010; Mednick et al., 2009; Ellenbogen et. al, 2006). These kinds of comparisons fail to control for the potential benefit of a period of quiet, wakeful rest (Wamsley, 2019). A growing body of literature suggests 
that short periods of unoccupied wakeful rest may facilitate memory consolidation in a manner similar to that proposed to occur during sleep (Brokaw, et al., 2016; Craig et al., 2018; Dewar et al., 2012; Gottselig et al., 2004; Humiston \& Wamsley, 2018).

The canonical wakeful rest paradigm consists of an encoding phase, an immediate retrieval phase, a wakeful rest or distractor phase, and a final delayed retrieval phase. The critical manipulation occurs during the period after the immediate retrieval test: participants either undergo $\sim 10$ minutes of wakeful rest, where they rest alone in a quiet room without access to electronics or reading materials or perform a simple distractor task (i.e., passive listening, visual search, etc.; Dewar et al., 2012; Millar \& Balota, 2018). Finally, participants complete a delayed retrieval test. Performance following the wakeful rest period is typically better than performance following the distractor task (see Wamsley, 2019 for a review) and it is hypothesized that wakeful rest may reduce forgetting by allowing for memory consolidation processes to occur (Dewar et al., 2009; Dewar et al., 2010; Dewar et al., 2012; Tambini et al., 2010; Wixted, 2004) ${ }^{1}$. The critical link to the present question regarding the functional role of MW is that the wakeful rest period, in which the participant is not given a task to direct attention, affords an excellent opportunity for MW.

Proponents of the wakeful rest literature also argue that it may be a highly desirable and applicable for use in populations susceptible to memory decline such as older adults and amnestic patients. Indeed, Craig and colleagues (2016) demonstrated that wakeful rest exhibited beneficial effects on cognitive map accuracy for both younger and older adults and Alber et al. (2014) showed that wakeful rest benefited both amnestic patients and healthy controls on prose

\footnotetext{
${ }^{1}$ One might argue that wakeful rest simply provides less interference than the distractor task. However, several pieces of literature have indicated that distractor tasks that do not engage episodic memory processes also allow for consolidation (see Varma et al., 2018; Varma et al., 2019).
} 
passage retention as compared to a spot-the-difference distractor task. Together, these results suggest that using a simple wakeful rest protocol may benefit memory retention not only in younger adults but also in populations vulnerable to memory decline. However, further work is necessary to replicate these findings using criterion tests other than cognitive maps and prose passages (see Millar \& Balota, 2018).

As noted earlier, periods of wakeful rest offer an excellent context for MW to occur. Song and Wang (2019) have demonstrated that MW most often occurs the in the absence of external demands or during low task engagement and, pertinent to the present hypothesis, that the contents of MW are predominantly episodic in nature (i.e., akin to replay). To better characterize the offline states under which wakeful rest benefits memory, Brokaw et al. (2016) conducted an EEG wakeful rest study with younger adult participants. Participants listened to a short story and were asked to immediately recall as many details as possible. Next, participants either rested or performed a distractor task (the Snood video game) for 15 minutes. After the retention interval, participants rated the proportion of the $15 \mathrm{~min}$ interval that they had spent engaged in various predefined categories of mental activity (including "thinking about the past," "imagining the future," "thinking about the short story", "thinking about staying still," "counting the time," "mind was blank," "meditating," "sleeping," "thinking about something else," and "other"). Finally, a delayed recall test was administered. The results indicated that the wakeful rest period enhanced memory for the short story as compared to the distractor task and that better memory was associated with (1) a specific slow-wave EEG signature associated with sleep and internal mentation and (2) increased episodic MW. The results of this study suggest that wakeful rest may benefit memory via a consolidation process supported by slow, oscillatory EEG activity 
and increased MW related to offline memory replay ${ }^{2}$. However, given the substantial variability in individuals' MW contents during wakeful rest periods (see Brokaw et al., 2016; Varma et al., 2019), one might wonder whether thoughts could be directly influenced to enhance memory reactivation during wakeful rest periods. Indeed, several targeted memory reactivation studies have investigated this issue.

Oudiette and colleagues (2013) used auditory cues to covertly reactivate recently encoded memory traces. Participants learned object-location associations which were paired with characteristic object sounds (i.e., a picture of a cat in a specific grid position paired with the sound of a "meow"; a number was also superimposed on each object to indicate the potential future reward for remembering its location). Next, participants were shown an object from encoding paired with the associated sound and asked to recall the grid location. Participants then underwent one of four between-subjects post-encoding delay conditions: sleep, sleep with cues, low-demand task, or low-demand task with cues. In the low-demand working memory task, participants were shown random numbers between 1 and 9 and asked to press a button if two consecutive numbers were even or odd. During the sleep with cues and low-demand task with cues conditions, several pre-selected sounds from the encoding period were played while participants slept or performed the low-demand task. The goal of presenting these auditory cues was to selectively reactivate the earlier memory traces and boost consolidation for a subset of the encoded object-location pairs. The results indicated that cued object-location pairs were recalled more accurately than the uncued object-location pairs with similar effect sizes in the sleep and awake conditions. The authors argued that this covert targeted reactivation of memories may aid in strengthening and stabilizing recently encoded information akin to the function of

\footnotetext{
${ }^{2}$ For further evidence linking slow, oscillatory EEG activity during wakefulness to memory consolidation see Wamsley and Summer (2020).
} 
consolidation/replay processes that occur during sleep (Oudiette et al., 2013). Although this interesting study has been cited over 200 times, it is somewhat limited by (1) the small sample size $(\mathrm{N}=15)$ for each between-participants condition, (2) the lack of thought probes during the awake conditions to directly attribute any benefits to offline memory replay, and, specifically given the goal of the current study, (3) the lack of an older adult sample to examine whether this targeted reactivation procedure may be useful for populations vulnerable to episodic memory decline.

\section{Aging and Mind-Wandering}

Although one would a priori predict that older adults would report more MW than younger adults, given that both MW and aging are associated with poor attentional control (Jackson \& Balota, 2012; Zacks \& Hasher, 1994), there is robust evidence indicating that older adults report less MW than younger adults (Frank et al., 2015; Giambra, 1973; Jackson \& Balota, 2012; Krawietz et al., 2012). Given that MW and episodic memory are similar in content, recruit overlapping neural regions, and both decline with age, it is possible that the robust episodic memory decline may in part reflect older adults' decreased likelihood to engage in MW and reactivate recently encoded information during periods of low demand.

Few studies in the extant literature have explored the possible benefits of MW in the context of aging and memory consolidation. However, one study by Varma et al. (2019) examined whether a brief 9-minute post-encoding period of wakeful rest may combat older adults' declines in episodic memory and to what extent MW propensity may serve a role in this memory decline. Older adult participants were first asked to study a list of picture-word pairs. Next, participants either performed a 2-back task or simply sat quietly in the testing room, assuming that MW would be more prevalent in the wakeful rest condition as compared to the 2- 
back condition. After the post-encoding delay period, participants' memory for the encoded picture-word pairs was tested using an old-new recognition memory test. Finally, participants completed a MW questionnaire which assessed daily life MW propensity (Imaginal Processes Inventory, MW subscale). Participants with greater MW propensity showed the largest benefit in memory performance following the rest period compared to the 2-back period. The authors argued that the degree to which episodic memory consolidation occurred during the postencoding wakeful rest period may depend on individual differences in MW propensity and that MW may assist in episodic memory formation and consolidation similar to memory reactivation during sleep (Varma et al., 2019).

Ultimately, both Brokaw and colleagues (2016) and Varma and colleagues (2019) demonstrated that improved memory following a period of wakeful rest is associated with decreased attention to the external environment and increased MW. Thus, it seems that MW may facilitate memory consolidation via mechanisms (possibly replay) similar to those that operate during sleep. Nevertheless, Brokaw et al. (2016) and Varma et al. (2019) are limited in several respects. First, neither study included both younger and older adults to compare memory performance and MW to address the issue of age-related changes in consolidation. Second, neither study included online thought probes, as commonly used in the MW literature, to more precisely characterize participants' thought contents during the wakeful rest and distractor intervals.

\section{The Current Study}

The literature reviewed above indicates that (1) memory consolidation can take place in offline states such as wakeful rest (Wamsley, 2019), (2) wakeful rest, which affords an opportunity for MW, is associated with improved episodic memory (Brokaw et al., 2016; Varma 
et al., 2019), (3) compared to younger adults, older adults show declines in episodic memory performance and report less MW, and (4) covert memory reactivation, via cueing, during offline states appears to promote consolidation targeted (cued) memories (Oudiette et al., 2013; Rasch et al., 2007). Given these observations, the present set of studies aimed to investigate whether MW may serve a functional role in memory consolidation by examining the relation between MW and episodic memory in younger and older adults.

In pursuit of these goals, the experimental design of the current set of studies addressed several critical methodological issues in the literature. First, no study to date has included both younger and older adults to better characterize the relationships among age, MW, and memory consolidation. Second, studies in the wakeful rest literature have avoided the use of online thought probes (presumably to avoid interrupting participants' wakeful rest) which are critical for better understanding participants' thought contents during the passive retention intervals. Third, there have not been any targeted memory reactivation studies which have compared younger and older adults, nor have there been any studies that have directly assessed the effect of memory cues on younger and older participants' ongoing thought contents. Fourth, no studies to date have investigated the influence of targeted memory reactivation on cued recall response latencies. As described below, the addition of a response latency measure was particularly important in this series of studies.

The present series of experiments addressed these issues by using a modified version of the Oudiette et al. (2019) paradigm, as shown in Figure 1. Both younger and older adults a) encoded associates (e.g., word-picture-sound triads), b) received targeted reactivation cues (e.g., the sound of a cow mooing) during a post-encoding retention interval filled with a low-demand "Shapes" task which has been shown to encourage MW, and c) were tested on their retrieval 
speed and accuracy for the cued and uncued stimuli from the initial encoding task. Additionally, in each experiment, thought probes were presented during the MW-inducing Shapes task to assess participants' thought contents. During Experiment 1, the paired associates were incidentally encoded, and auditory cues were presented during the Shapes task. Because of the intriguing cuing effects observed in the response latencies in Experiment 1, an attempt was made to replicate and extend this pattern. In the second experiment, as in Oudiette et al. (2019), participants were incentivized via monetary rewards based on their memory performance. In Experiment 3, picture cues instead of auditory cues were presented in an attempt to produce a more robust cueing effect.

\section{Experiment 1}

\section{Method}

\section{Participants}

Participants across all studies were tested online and recruited using Prolific (prolific.co) and compensated at a rate of $\$ 6.50 /$ hour. Inclusion criteria for all studies included native English-speaking ability, normal or corrected-to-normal vision, and normal hearing. Participants were not allowed to participate in more than one of the present studies. All experiments and procedures were approved by the Washington University in St. Louis Institutional Review Board.

Sixty younger and 60 older adults met inclusion criteria and were recruited to participate in Experiment 1; however, two older adults failed to respond to any of the thought probes. Given the possibility that these participants either did not understand the instructions or were not engaged in the task, these two older adults were removed before analysis. The data from four older adult participants were replaced because they reported writing information down during the 
experiment. Therefore, the analyses reported include 60 younger adults and 58 older adults. Table 1 displays the age ranges and performance on the Wechsler Memory Scale (WMS; Wechsler \& Stone, 1973) for both the younger and older adults across experiments.

\section{Materials}

Stimuli consisted of a set of 52 picture-sound-word triads. Pictures and sounds were drawn from Oudiette et al. (2013) and words were drawn from Varma et al. (2019). Pictures were of everyday, concrete objects. Each picture was accompanied by a characteristic sound (e.g., dog and "woof", bell and "clang"). The word stimuli consisted of adjectives that ranged from three to nine letters in length $(\mathrm{M}=6.06$ letters). To ensure that the picture-word pairs were unrelated, the triads were pseudorandomly combined such that pairs with any obvious preexisting association were re-paired until all adjectives were paired with an object/sound which did not have any clear relationship. For counterbalancing purposes, the 52 triads were rotated through two different lists (i.e., cued and uncued) such that half of the triads were cued for a given participant and half were uncued and that across participants each pair occurred equally in the cued and uncued conditions.

The experimental programs were written in PsychoPy (Pierce et al., 2019) and hosted on Pavlovia (pavlovia.org). Manual responses were made using participants' keyboards. Participants were required to complete the experiment on a desktop or laptop computer. If a participant attempted to begin the experiment on a mobile device, tablet, or other device, the program would error out and send the participant back to the Prolific study screen.

\section{Procedure}

Before beginning the experiment, participants received an auditory check with the following instructions: "This is an audio check. Please follow the instructions being presented 
auditorily. If you cannot hear the audio check, you will not be able to complete this experiment. Please close the tab." Participants then received auditory instructions stating: "This is an audio check. Please take a moment to adjust the volume to a comfortable setting. Once the experiment begins, please refrain from adjusting the volume. This is critical for the experiment. If you can hear this message at a comfortable volume, please press the ' $Q$ ' key on your keyboard to move on." These instructions repeated (up to three times) until the participant responded with the correct key response (pressing Q). If the participant pressed Q, the experiment would commence. If the participant did not press Q before the auditory instructions had repeated three times, the experiment would end.

As described earlier and shown in Figure 1, each participant performed three tasks during the experiment. First, participants performed an encoding task. During each encoding trial, participants were asked to create an imaginative association between the word (e.g., "colorful") and the picture-sound pair (e.g., a picture of a cow paired with the sound of a "moo"). The word was presented on the screen for a fixed duration of 1 second before the picture and sound were presented along with the word for a fixed duration of 4 seconds. Each sound was 1 second in length and each sound was played three times in a row to ensure encoding. Thus, the sound played for 3 seconds. Then, participants were asked to rate the vividness of their imagined association on a scale of 1-5 using the keyboard within the next 5 seconds. After 5 seconds had elapsed, the next trial began with a fixation cross displayed for 0.5 seconds. This was kept constant regardless of when participants responded. In order to ensure sufficient encoding, the encoding list was presented twice.

Next, participants performed a modified version of the Shapes task adapted from O’Callaghan et al. (2015) to examine MW. Participants were presented with instructions that 
read: "This portion of the study is looking at relaxation. Please look at the computer screen and try to relax with your eyes open while attending to the shapes." This screen was displayed until participants pressed the spacebar to advance. Participants were then informed about the thought probes with the following instructions: "Throughout this task, you will also be asked about your thoughts. You will see the following: What were you thinking about immediately before this screen? (1) The previous task (thinking about the pictures, sounds, and words), (2) The current task (thinking about the shapes), (3) Unrelated to the experiment (thinking about something not related to the experiment). Please press the number key which corresponds with your response $(1,2$, or 3$)$. Press SPACE when you are ready to begin this task." This screen was presented until participants pressed the spacebar to continue with the experiment. Participants were given up to $10 \mathrm{sec}$ to respond to each thought probe. In addition to the thought probes, auditory sound cues were played pseudorandomly throughout the duration of the Shapes task. Similar to encoding, the auditory cues were played for a duration of 3 seconds such that each 1 -second sound was repeated three times consecutively. Specifically, thought probes and auditory cues were presented in a pseudorandomized order such that thought probes were presented a minimum of 6 seconds, a maximum of 42 seconds, and on average 24 seconds between each other. Auditory cues were presented a minimum of 6 seconds, a maximum of 45 seconds, and on average 20 seconds between one another. Thought probes and auditory cues were spaced a minimum of 3 seconds, a maximum of 36 seconds, and on average 10.94 seconds between one another. The modified Shapes task lasted for approximately 10 minutes. During this time, 26 cues (i.e., half of the sounds from encoding) were played and 20 thought probes were presented.

After the Shapes task, participants performed a speeded cued recall task of the initially encoded picture/sound adjective pairs. Participants were presented with instructions that read: 
"You will be presented with a sound and picture from earlier in the experiment. Please recall the WORD that was paired with the sound and picture from earlier in the experiment. Please type your response using the keyboard and press ENTER after you are done to move on. Please do this as quickly and accurately as possible. Press SPACE when you are ready to begin this task." This screen was presented until participants pressed the spacebar to advance. After typing their response and pressing the return key or 10 seconds expired, whichever came first, the next trial began with a fixation cross displayed for 0.5 seconds $^{3}$.

After the cued recall task, participants performed the 10 item WMS Paired Associates Task and then answered several questions about their experience during the experiment. During encoding each paired associate was presented for 3 seconds, and during each recall trial, participants were given a maximum of 10 seconds to respond.

After the WMS, all participants completed a short questionnaire. Participants were asked three yes/no questions: (1) "Did you try to remember the pictures and words that were paired with the sounds when the sounds were played during the shapes task?", (2) "Were there any distractions in your environment (i.e., other tabs open, music playing, TV or other people speaking in the room)?", and (3) "Please be honest: did you write anything down (i.e., for the memory tasks) during this experiment? Your response will not affect your compensation.” These data are provided in Appendix A in the Supplemental Materials.

Older adult participants were also asked several questions about their hearing. These questions included: (1) “Do you wear a hearing aid?” (Yes / No), (2) “On a scale from 1-5, how much trouble do you have hearing during conversations?" with 1 being "No Trouble At All" and

\footnotetext{
${ }^{3}$ To confirm that participants' spelling did not influence the findings, an analysis was conducted on the data for Experiments 1 and 2 which used a strict spelling correction. This did not change any of the results reported in either experiment.
} 
5 being “A Lot Of Trouble”, (3) "Do you require subtitles to watch TV?” (Yes / No), and (4)

"On a scale from 1-5, how well could you identify each of the sounds as they were presented?" with 1 being "Could NOT Identify Any” and 5 being “Could Identify ALL." These data are provided in Appendix B in the Supplemental Materials. All data, code, and study materials are available upon request.

\section{Results}

For all results reported, statistical significance was set at $p<.05$, two-tailed test, unless otherwise noted. Effect sizes of eta squared $\left(\eta^{2}\right.$, Olejnik \& Algina, 2003) are reported for significant $F$ tests and Cohen's $d$ ( $d$, Cohen, 1988) for significant $t$-tests. Adjusted degrees of freedom are reported such that unequal variances were assumed and the Welsh approximation was applied.

Response latencies were defined by the initial key press after the picture object was presented during cued recall. Any response times less than three standard deviations below or greater than three standard deviations above the individual's mean response time were removed. This trimming procedure eliminated $1.54 \%$ of trials for younger adults and $1.42 \%$ of trials for older adults in Experiment 1. After this trimming procedure, general slowing was corrected for by z-scoring response times within each participant based on that participant's mean and standard deviation. This transformation controls for age-related differences in response time and variability (see Faust, Balota, Spieler, \& Ferraro, 1999), and allows one to examine effects of a variable (in this case, cueing) on response times above and beyond scaling differences.

First, analyses on recall accuracy are presented to examine if cued stimuli were more accurately recalled, compared to uncued stimuli. Second, correct recall standardized response 
time results are presented to examine if there is a cuing effect on correct retrieval latencies ${ }^{4}$. For both accuracy and standardized response times, as mentioned earlier, it was hypothesized that if reductions in MW propensity with age are associated with age-related declines in episodic memory, then younger, but not older, adults should show a recall benefit for cued stimuli as compared to uncued stimuli since older adults are less likely to engage in MW episodes that may be important for memory consolidation processes. Finally, the frequency of thought probe responses and their relationship to episodic memory performance was examined to test the hypothesis that participants' mental states during the Shapes task would predict memory performance.

Recall Accuracy. Percent correct recall accuracy as a function of Age Group and Cue for Experiment 1 are displayed on the left in Figure 2. Overall, younger adults correctly recalled more words than older adults; however, cued stimuli were not recalled better than uncued stimuli. These patterns were confirmed by a two-way Age Group (young, old) by Cue (uncued, cued) mixed-factor analysis of variance (ANOVA) which yielded a main effect of Age Group, $F(1,116)=6.89, p=.01, \eta^{2}=.05$. Neither the main effect of Cue, $F(1,116)=0.51$, nor the Age Group by Cue interaction, $F(1,116)=0.36$, reached significance, $p \mathrm{~s}>.35$. Follow-up $t$-tests further confirmed that neither age group showed a benefit in accuracy for the cued stimuli, $p$ s $>$ .25 .

Correct Recall Standardized Response Times. Correct recall standardized response times for Experiment 1 are displayed on the left in Figure 3. As shown, the results indicated that there was a cuing effect for the younger adults, but not for the older adults. This pattern was confirmed by a two-way Age Group (young, old) by Cue (uncued, cued) mixed-factor ANOVA,

\footnotetext{
${ }^{4}$ Raw, mean response latencies as a function of Age Group, Cue, and Experiment are presented in Appendix C in the Supplemental Materials.
} 
which yielded a marginal main effect of Cue, $F(1,116)=2.89, p=.09, \eta^{2}=.01$, and a reliable Age Group by Cue interaction, $F(1,116)=5.38, p=.02, \eta^{2}=.02$. Follow-up $t$-tests indicated that younger adults produced a reliable cueing effect in standardized response latencies, $t(56)=$ 2.23, $p=.03, d=0.55$, but this effect did not approach significance for older adults, $t(56)=0.33$, $p=.74$

Thought Probe Responses. Thought probe response percentages for Experiment 1 as a function of Age Group and Experiment are displayed in the first row of Table 2. As expected, older adults reported less MW than younger adults. This was reflected in older adults reporting numerically more on task thoughts and significantly less experiment-unrelated MW than younger adults. However, it is noteworthy that younger and older adults did not differ in their memory task-related MW frequency.

The relationship between participants' thought probe responses and the degree to which they benefited from the cues presented during the Shapes task during cued retrieval was also examined. As shown in Figure 4, participants who reported more memory task-related MW during the Shapes task showed a larger accuracy cueing effect at recall, $r(116)=.21, p=.02$. Furthermore, there was also evidence that the less participants thought about the Shapes task itself (fewer on task reports), the larger accuracy cueing effect they produced at retrieval, $r(116)$ $=-.20, p=.03$. Both correlations remained significant after controlling for age, $p \mathrm{~s}<.03$. Interestingly, there were no reliable correlations with the standardized response time cueing effects, $p \mathrm{~s}>.49$.

Self-Reported Hearing. An analysis was conducted to examine the possibility that the results from Experiment 1 were influenced by age-related declines in hearing. Several pieces of evidence suggest that this is not likely the case. First, using the Prolific online recruitment 
system, only participants who had indicated they had normal or corrected-to-normal hearing received the advertisement for the study. Second, participants had to pass the auditory check, described above, in order to begin the experiment. Third, as mentioned in the methods section, at the end of the experiment, older adults were asked several questions about their hearing including (1) whether they wear a hearing aid, (2) how much trouble they have hearing during conversations, (3) if they require subtitles to watch TV, and (4) how well they could identify the experimental sounds. Only two of the 60 older adults in Experiment 1 indicated that they reported wearing a hearing aid and none reported requiring subtitles to watch TV. Importantly, one can examine whether older adults' self-reported hearing difficulties (questions 2 and 4) carried any power in predicting participants' thought probe responses or cueing effects. Neither older adults' self-reported difficulty hearing during conversations nor their self-reported difficulty identifying the experimental sounds were significantly correlated with their thought probe responses or their cueing effects, $p \mathrm{~s}>.25$.

\section{Discussion}

Experiment 1 provided initial support for the hypothesis that reductions in MW propensity may be associated with age-related declines in episodic memory due to differences in younger and older adults' reactivation of cued traces during the Shapes task. The main results of Experiment 1 indicated that younger adults showed both increased MW during the Shapes task and better episodic memory during the cued recall task as compared to older adults. Furthermore, the results from the standardized response latency analyses indicated that younger adults produced a greater benefit from the auditory cues presented during the Shapes task compared to when they had not been cued and as compared to older adults. This is consistent with the primary hypothesis that younger adults would be more sensitive to the targeted memory reactivation cues 
presented during the Shapes task than older adults. Furthermore, participants who reported more memory task-related MW during the Shapes task showed a larger accuracy cueing effect and participants who reported more MW overall showed a larger cueing effect at retrieval. This pattern did not change when age was covaried out of the analyses. Surprisingly, although there was a clear effect of cueing on younger adults' response latencies, there was no cueing effect present in recall accuracy for either younger or older adults.

\section{Experiment 2}

Although Experiment 1 provided some evidence for the original hypothesis, it is unclear why there was no cuing effect in recall accuracy. In light of this, it is noteworthy that Oudiette et al. (2013) incentivized participants by superimposing a value on each object at encoding to indicate the potential future payoff for remembering its location. Indeed, they argued that although it is possible that memory replay occurs randomly and with equal likelihood for all recently acquired memories, there is also evidence that memory replay may be driven by preferential consolidation (Lansink et al., 2008, 2009; Oudiette et al., 2013). Evidence of preferential consolidation comes from studies indicating that emotional salience (Hu et al., 2006; Sterpenich et al., 2009), intention to remember (van Dongen et al., 2012; Wilhelm et al., 2011), and anticipation of a future reward for correct remembering (Fischer \& Born, 2009) all enhance consolidation during sleep. These studies support the notion that memory consolidation entails some selectivity for more valuable information and may be an indirect consequence of the downscaling of synaptic connectivity which occurs during consolidation (Tononi \& Cirelli, 2006). Thus, similar to Oudiette et al. (2019) and to better motivate the encoding, reactivation/consolidation, and retrieval of the experimental stimuli, participants in Experiment 2 were provided with performance-based incentive instructions at the beginning of the study. 


\section{Method}

\section{Participants}

Sixty younger and 60 older adults met inclusion criteria and were recruited to participate in Experiment 2; however, two of the younger adults failed to respond to any of the thought probes and three younger adults failed to correctly recall any stimuli. The data from six younger and seven older adult participants were replaced because they reported writing information down during the experiment. Therefore, the analyses reported include 55 younger adults and 60 older adults.

\section{Materials}

The materials used in Experiment 2 were the same as those used in Experiment 1.

\section{Procedure}

The procedure was the same as the procedure used in Experiment 1 with the exception that, after completing the auditory check, participants received the following incentive instructions: "In this experiment, you will first study sound-picture-word triads. Later in the experiment, you will be asked to remember which word was paired with each picture/sound. If you recall more than $40 \%$ of the words correctly, you will receive an additional monetary bonus! You will receive an additional 5 cents per word you correctly recall past the $40 \%$ accuracy mark. This means you should do your best to remember the sound-picture-word triads in the first part of the experiment and recall them to the best of your ability in the memory portion of the experiment. Press the SPACEBAR when you are ready to continue." These instructions were displayed until participants pressed the spacebar to continue.

\section{Results}

The response time trimming procedure described earlier eliminated $2.05 \%$ of trials for younger adults and $1.15 \%$ of trials for older adults in Experiment 2. 
Recall Accuracy. Percent correct recall as a function of Age Group and Cue, for Experiment 2, is displayed in the middle of Figure 2. Overall, younger adults again correctly recalled more words than older adults and cued stimuli were recalled slightly better than uncued stimuli. These patterns were examined using a two-way Age Group (young, old) by Cue (uncued, cued) mixed-factor ANOVA, which yielded a main effect of Age Group, $F(1,113)=$ $65.36, p<.001, \eta^{2}=.35$. However, neither the main effect of Cue, $F(1,113)=2.12, p=.15$, nor the Age Group by Cue interaction approached significance, $F(1,113)=0.36, p=.55$. Neither younger nor older adults produced a reliable accuracy cueing effect, $p \mathrm{~s}>.19$.

Correct Recall Standardized Response Times. Correct recall standardized response times are displayed in the middle of Figure 3. Replicating Experiment 1, younger adults again recalled the cued stimuli more quickly than uncued stimuli, whereas this was not the case for older adults. These patterns were confirmed by a two-way Age Group (young, old) by Cue (uncued, cued) mixed-factor ANOVA, which yielded a main effect of Cue, $F(1,113)=12.68, p$ $<.001, \eta^{2}=.05$, but no effect of Age, $F(1,113)=0.07, p=0.79$. The Age Group by Cue interaction approached significance, $F(1,113)=2.51, p=.11$. Planned $t$-tests indicated that, as in Experiment 1, the younger adults produced a reliable cueing effect in standardized response latencies, $t(52)=4.34, p<.001, d=1.06$, but this did not approach significance for older adults, $t(55)=0.86, p=.39$

Thought Probe Responses. Thought probe response percentages for Experiment 2 are displayed in the second row of Table 2. Older adults again reported less MW than younger adults. Specifically, older adults reported significantly more on task thoughts and, in this case, less memory task-related MW than younger adults. Younger adults only reported marginally more thoughts unrelated to the experiment as compared to older adults. 
The relationship between memory task-related MW during the Shapes task and the degree to which participants benefited from the cues presented during the Shapes task at retrieval was again examined. As shown in Figure 5, participants who reported more memory task-related MW during the Shapes task showed larger cueing effects in correct standardized response times, $r(107)=.16, p=.10$, although this relationship did not reach significance. Furthermore, there was also evidence that the less participants thought about the Shapes task itself (fewer on task reports), the larger cueing effect they produced at retrieval (again, in correct standardized response times), $r(107)=-.19, p=.04$. After controlling for age, however, this correlation was only marginal, $p=.09$.

Self-Reported Hearing. Again, the possibility that the results could be influenced by age-related declines in hearing was examined. The same analyses as in Experiment 1 were conducted to test for any possible relationship between older adults' self-reported hearing difficulties and their performance in the cued recall task. Neither older adults' self-reported difficulty hearing during conversations nor their self-reported difficulty identifying the cued sounds was significantly correlated with either their thought probe responses or their cueing effects, $p s>$.11. Furthermore, even when collapsing across Experiments 1 and 2, neither older adults' self-reported hearing difficulty during conversations nor their self-reported difficulty identifying the experimental sounds significantly predicted their cueing effects, $p s>.11$.

\section{Discussion}

Experiment 2 again provided support for the hypothesis that reductions in MW propensity may be associated with age-related declines in episodic memory due to differences in younger and older adults' ability to reactivate cued traces during MW. The presence of an incentive in Experiment 2 did increase overall recall performance compared to Experiment 1, 
and this occurred primarily for younger adults, $t(111.9)=2.48, p=.01, d=0.46$, but not for older adults, $t(115.6)=1.65, p=.10$. It is likely that monetary incentives are more important for younger adults than older adults or that older adults were already performing at their maximum level in Experiment 1 (without the incentive). More importantly, for the present hypotheses, the results from Experiment 2 again indicated that younger adults produced a robust benefit from the auditory cues in the standardized response latencies. However, this effect was not present for the older adults. This pattern clearly replicates the results from Experiment 1. Finally, participants who reported more MW overall showed a larger cueing effect at retrieval supporting the functional role of MW on episodic memory.

\section{Experiment 3}

Although there was a clear effect of cue on standardized response latencies in the younger, but not older, adults in both Experiments 1 and 2, it is surprising that there was still no effect of cue on recall accuracy. This lack of effect of cue on recall accuracy limits the ability to more robustly test age-related differences. Therefore, Experiment 3 aimed to increase the effectiveness of the targeted reactivation cues in two ways: First, it is possible that the complexity of the sound/picture/word triad made it difficult to develop a well-integrated memory trace during encoding. Thus, in Experiment 3, all auditory information was omitted, and hence, participants encoded simple picture-word pairs. Second, during the Shapes task, picture cues were re-presented to reactivate the earlier picture-word stimuli. The hypothesis tested in Experiment 3 was that the picture cues would be more salient and more effectively reactivate the paired adjective than the sound cues. Thus, the main goal of Experiment 3 was to increase the influence of the cues on recall accuracy to afford a more powerful test of any age-related differences. 


\section{Method}

\section{Participants}

Sixty younger and 60 older adults met inclusion criteria and were recruited to participate in Experiment 3; however, because of concerns regarding task engagement, four younger adults and two older adults who failed to respond to any of the thought probes and two younger adults and two older adults who failed to correctly recall any stimuli were removed before analysis. The data from six younger and five older adult participants were replaced because they reported writing information down during the experiment. Therefore, the analyses reported include 54 younger adults and 56 older adults.

\section{Materials}

The materials were the same as those used in Experiment 2, with the elimination of the auditory cues.

\section{Procedure}

The procedure was the same as described in Experiment 2 with the following changes: First, there was no auditory check at the beginning of the experiment because no auditory stimuli were presented in Experiment 3. Instead of the auditory check instructions, participants were presented with a screen in its place which read: "Please take a moment to silence any external distractions and mute any background tabs. Please press the spacebar to continue." Second, as noted, during the encoding task, participants were simply asked to generate, and then rate the vividness of, an imaginative association between a word and picture (as opposed to the picture/sound/word triad in the previous experiments). Third, during the Shapes task, participants were presented with picture cues, as compared to the auditory cues used in Experiments 1 and 2. Picture cues were presented using the same pseudorandomization procedure described in 
Experiment 2 and presented for the same duration as the auditory cues were presented in Experiments 1 and 2 (i.e., 3 seconds). Fourth, during the cued recall task, rather than the instructions described in Experiment 2, which referenced encoded sounds, participants were presented with instructions that read: "You will be presented with a picture from earlier in the experiment. Please recall the WORD that was paired with the picture from earlier in the experiment. Please type your response using the keyboard and press ENTER after you are done to move on. Please do this as quickly and accurately as possible." Last, although recruitment and inclusion criteria for Experiment 3 were the same as the previous experiments and there were no auditory stimuli presented, older adult participants were still asked to complete a modified version of the post-experiment hearing questionnaire which included the following questions: (1) "Do you wear a hearing aid?" (Yes / No), (2) “On a scale from 1-5, how much trouble do you have hearing during conversations?" with 1 being "No Trouble At All" and 5 being "A Lot Of Trouble", (3) "Do you require subtitles to watch TV?" (Yes / No), and (4) "On a scale from 1-5, how well could you identify each of the pictures as they were presented?" with 1 being "Could NOT Identify Any" and 5 being "Could Identify ALL."

\section{Results}

The response time trimming procedure described earlier eliminated $1.79 \%$ of trials for younger adults and $1.74 \%$ of trials for older adults in Experiment 3.

Recall Accuracy. Percent correct recall for Experiment 3 is displayed as a function of Age Group and Cue on the right in Figure 2. Overall, younger adults correctly recalled more words than older adults and cued stimuli were recalled better than uncued stimuli. These patterns were confirmed by a two-way Age Group (young, old) by Cue (uncued, cued) mixed-factor ANOVA, which yielded a main effect of Age Group, $F(1,108)=28.25, p<.001, \eta^{2}=.20$, a 
main effect of Cue, $F(1,108)=5.43, p=.02, \eta^{2}=.002$, and an Age Group by Cue interaction, $F(1,108)=4.67, p=.03, \eta^{2}=.002$. In contrast to the previous experiments, planned $t$-tests indicated that older adults produced a reliable accuracy cueing effect, $t(55)=3.01, p=.004, d=$ 0.17 , but this did not approach significance for younger adults, $t(53)=0.09, p=.91$.

Correct Recall Standardized Response Times. Correct recall standardized response times for Experiment 3 are displayed on the right in Figure 3. Overall, participants were able to correctly recall cued stimuli more quickly than uncued stimuli. Furthermore, the results provided support for the hypothesis that increasing the cue salience allowed both younger and older adults to make use of the cues to trigger reactivation of recently encoded information and promote consolidation during MW such that both age groups correctly recalled cued stimuli more quickly than uncued stimuli. These patterns were confirmed by a two-way Age Group (young, old) by Cue (uncued, cued) mixed-factor ANOVA, which yielded a robust main effect of Cue, $F(1,108)$ $=67.90, p<.001, \eta^{2}=.24$. Neither the main effect of Age Group nor the Age Group by Cue interaction approached significance, $p \mathrm{~s}>.20$.

Thought Probe Responses. Thought probe response percentages are displayed in Table 2. Older adults again reported being more on task during the Shapes task than younger adults. As shown, these patterns were confirmed with $t$-tests which indicated that older adults reported significantly more on task thoughts and less MW than younger adults. Although older adults produced less experiment-unrelated MW than younger adults, there was no effect of age on memory task-related MW.

The relationship between participants' thought probe responses and the degree to which they benefited from the cues presented during the Shapes task later on at retrieval was again examined. Interestingly, in contrast to the previous experiments, there were no significant 
relationships between participants' thought probe responses during the Shapes task and their cueing effects produced at recall, $p \mathrm{~s}>.10$.

\section{Discussion}

There are several key findings from Experiment 3. There was again only a relatively small effect of cue on recall accuracy $(2.48 \%)$, and this effect was only reliable for older adults. Thus, although overall performance was higher in Experiment 3 as compared to Experiment 2, as confirmed by a three-way Age Group (young, old) by Experiment (Experiment 2, Experiment 3) by Cue (uncued, cued) mixed-factor ANOVA which yielded a significant main effect of Experiment, $F(1,221)=16.13, p<.001, \eta^{2}=.06$, increasing the salience of the cue and decreasing the complexity of the encoded stimuli only produced a small accuracy cuing effect for the older adults. Importantly, however, neither the Age by Cue, Experiment by Cue, nor Age by Experiment by Cue interactions approached significance, $p \mathrm{~s}>.05$. The inconsistency of the cueing effect in recall accuracy across experiments is quite noticeable.

The results from Experiment 3 produced a much more robust pattern in the standardized response time data. Specifically, Experiment 3 replicated the strong cueing effect in standardized response latencies for young adults observed in Experiments 1 and 2, and importantly also produced a cuing effect for older adults. Indeed, the three-way Age Group (young, old) by Experiment (Experiment 2, Experiment 3) by Cue (uncued, cued) mixed-factor ANOVA yielded a significant Age Group by Experiment by Cue interaction, $F(1,221)=8.17, p=.004, \eta^{2}=.02$, reflecting the increased cue effect especially for the older adults in Experiment 3, compared to Experiment 2.

\section{General Discussion}


The primary aim of the present set of studies was to investigate the nature of the relationship between MW and episodic memory in the context of healthy aging. The present studies explored two main questions: (1) Does MW serves a functional role, specifically in memory consolidation via post-encoding reactivation during offline states; (2) Are there age differences in the ability to reactivate episodic memories during MW and does this contribute to age-related declines in episodic memory? Before turning to discussion of the results, it is important to summarize the main findings.

Experiment 1 provided support for the hypothesis that reductions in MW propensity may be associated with age-related declines in episodic memory due to differences in younger and older adults' ability to reactivate cued traces during MW episodes. Specifically, there was a reliable Age Group by Cue interaction which indicated that younger adults produced facilitation in standardized correct recall response latencies for stimuli that were auditorily cued during the Shapes task, but there was no influence of the cues in the older adults. These results were interpreted as an indication that the auditory cues served to reactivate the picture/sound connection to the paired word, and that older adults were less likely to reactivate this connection than younger adults during the Shapes task. Furthermore, overall, participants who reported more memory task-related MW during the Shapes task showed a larger cueing effect, which suggests that the cues modulated participants' MW contents and in turn influenced their memory performance. However, surprisingly, there was no cueing effect in recall accuracy for either the young or older adults.

In Experiment 2, as in Oudiette et al. (2013), participants were incentivized via monetary rewards based on their memory performance to potentially increase the importance of the cues. Indeed, the addition of the incentive instructions boosted younger, but not older, adults' overall 
recall performance, possibly due to the older adults being more financially stable and/or older adults already being highly motivated (see Nicosia \& Balota, 2021). More importantly, the results again indicated that younger adults produced a robust benefit from the auditory cues in the standardized response latencies whereas there was no effect for older adults, replicating the findings observed in Experiment 1. There was no effect of cue in overall cued recall accuracy. These results were again consistent with the hypothesis that there is an age difference in the benefit from cues presented during the Shapes task to reinstate the picture/sound connection with the paired word, which facilitates retrieval speed. There was again a relationship between participants' MW contents and the magnitude of the cueing effect. Specifically, participants who reported more MW overall showed larger cueing effects.

In Experiment 3, the salience of the targeted memory reactivation cues was further increased by eliminating the auditory cues, thereby decreasing the complexity of the tripartite memory trace (i.e., sound/picture-word) to a paired associate (picture-word) and re-presenting the pictures as cues during the Shapes task. With these modifications, Experiment 3 yielded a robust cueing effect in standardized response times for both younger and older adults. Hence, increasing the salience of the retrieval cue during the Shapes task was sufficient to facilitate the speed to retrieve the target even in older adults. Second, we also obtained a relatively small effect of cue on recall accuracy, though it was only significant for the older adults.

The observed age differences in standardized response time cuing effects have been taken as evidence that older adults may be less likely to engage in memory task-related MW than younger adults, thereby decreasing their sensitivity to the cues during the shapes task. Indeed, every experiment indicated that older adults reported being more on task (and MW less) during the Shapes task than younger adults, as shown in Table 2. However, when one considers the 
particular type of MW, specifically memory task-related MW, younger and older adults report relatively similar frequencies. Older adults' decreased MW, compared to younger adults, occurred primarily in their reports of MW unrelated to the current experiment. Thus, it appears that age differences in the cuing effect were not due to younger adults thinking more about the specific list items, at least as measured by the current paradigm, but rather may reflect reactivation of these items during the Shapes task.

Across experiments, these results provide support for the Mildner and Tamir (2019) hypothesis that reactivation of encoded information during MW may serve as a mechanism that contributes to memory consolidation. Furthermore, at least with the auditory cues and the tripartite stimulus complex in Experiment 1, older adults were less likely to successfully reactivate encoded information as compared to younger adults, and this may have potentially contributed to their decreased episodic memory. The results from the first two experiments were interpreted within a retrieval facilitation framework wherein the cues serve to reactivate the earlier traces, and this reactivation benefitted retrieval speed at recall for the cued stimuli as compared to the uncued stimuli.

However, it is important to note an alternative interpretation of the consistent effect of cuing on the standardized response latencies. Specifically, one could argue that the cues produced a type of implicit priming due to repetition and hence increased the speed to recognize the cues during the memory test across experiments. This alternative account is contrasted with the hypothesized account which suggests that the cues presented during the Shapes task reactivate the trace from encoding, initiate replay of the trace, and thereby "grease the pathway" for faster retrieval. 
There are several pieces of evidence, which speak against the repetition priming account: First, there is considerable evidence that older adults produce robust repetition priming and familiarity-based effects, compared to younger adults (see, for example, Balota et al., 2000; Millar et al., 2017). If the cuing effects were primarily due to repetition priming, then one would expect older adults to produce a cueing effect in response latencies. However, as shown here, this was not the case.

Second, collapsing across the recall experiments which included cues during the Shapes task (i.e., Experiments 1, 2, and 3), there were significant correlations between memory taskrelated MW and several episodic memory performance measures, in addition to the magnitude of participants' cueing effects. Specifically, as shown in Appendix D in the Supplemental Materials, memory task-related MW during the Shapes task was significantly correlated with the magnitude of the accuracy cueing effect, $r(341)=.11, p=.03$, total recall accuracy, $r(341)=.15$, $p=.007$, and, importantly, participants' WMS accuracy, $r(341)=.12, p=.03$. The correlation between memory task-related MW during the Shapes task and WMS accuracy is particularly important because it suggests that memory task-related MW during the Shapes task is associated with episodic memory performance for an unrelated task and unrelated set of stimuli (i.e., WMS $)^{5}$. Specifically, if it were the case that participants were simply faster to respond at retrieval to cued stimuli as compared to uncued stimuli because of increased fluency with the cued object, then one would not expect a relationship between memory task-related MW and episodic memory performance on an unrelated task.

A residual issue not addressed by the present set of studies, however, is whether replay via targeted reactivation is an explicit or implicit (i.e., conscious or unconscious) process. As

\footnotetext{
${ }^{5}$ Furthermore, these correlations persisted after controlling for age.
} 
described in the Introduction, hippocampal replay studies have focused on observing cellular replay in rodents and hippocampal reactivation in humans. To date, these studies have not provided any insights regarding the thought contents (or level of awareness) that accompany these brain states. The current study aimed to fill this gap in the literature by including targeted reactivation cues coupled with thought probes. Although these effects appear to be more explicit than implicit in nature, it is unclear under which circumstances, and to what degree of awareness, reactivation may occur in more general, everyday circumstances when participants are not delivered cues and asked to report on their thought contents.

\section{Theoretical Implications}

The present studies are the first of their kind to directly test theoretical accounts which have arisen within the past few years. For example, Mildner and Tamir (2019) suggested that spontaneous thought (i.e., MW) may serve as an "unconstrained memory process." The authors argued that memory and spontaneous thought are similar in terms of their contents and neural mechanisms and that the "mental infrastructure that supports memory also gives rise to spontaneous thought." Not only were we able to directly measure whether the contents of participants' MW can benefit retrieval, but also that age differences in episodic memory, and its “infrastructure," modulate this ability at least to some extent.

Additionally, the fact that retrieval speed was more sensitive than accuracy to cueing effects in the present paradigm provides further insight into the nature of this reactivation. Because offline replay appears to benefit retrieval speed more so than accuracy, and younger adults produced a larger response time cueing effect than older adults, the implication here is that not only do age differences in episodic memory contribute to MW content and propensity, but participants' ability to MW about episodic events further contributes to downstream effects on 
memory consolidation and retrieval. The theoretical interpretation here is that because of their shared mechanistic underpinnings, the relationship between episodic memory and MW may be synergistic, such that episodic memory drives the frequency and contents of MW, and MW may in turn influence episodic memory retrieval speed.

As mentioned above and as shown in Appendix D in the Supplemental Materials, not only was MW correlated with cueing effects, but it was also significantly correlated with performance on the unrelated WMS test. This speaks to the notion of "shared infrastructure" between episodic memory and MW such that, although one might expect a relationship between MW and cueing effects due to the replay hypothesis, an overlap in underlying mechanisms would be necessary to explain the correlations between MW in the Shapes task and accuracy in an unrelated episodic memory test ${ }^{6}$. Ultimately, these results provide initial empirical support for the theoretical hypothesis put forth by Mildner and Tamir (2019) that, not only are episodic memory and MW associated with one another, but that they share a two-way street: episodic memory traces feed MW frequency and content, and MW drives offline, awake consolidation which can benefit later retrieval.

An intriguing observation in the present results was that retrieval speed produced a more robust cueing effect than accuracy. This may suggest that in order for offline reactivation to be beneficial, it may be necessary for the traces to be sufficiently encoded. Indeed, it may be the case that the cues during the Shapes task could only be reactivated and facilitate retrieval for traces that were already accessible. In contrast, for traces that are not well-established, offline replay triggered by the cues did not appear to be capable of reactivating the target (i.e., accuracy was not increased for information that was not initially fully encoded). This would also explain

\footnotetext{
${ }^{6}$ This claim is further bolstered by the results conducted in a pilot analysis which showed small but significant relationships between MW, an unrelated episodic memory composite score, and hippocampal and MTL volumes.
} 
why one observes a larger standardized response time cueing effect for younger adults as compared to older adults. Indeed, if older adults are less successful at producing a robust trace at encoding, as evidenced by their lower overall recall performance, they should be less likely to benefit from the reactivation cues, compared to the younger adults who appear to produce more robust episodic traces. Ultimately, it is possible that age differences in episodic memory contribute to MW content and propensity, and participants' MW about episodic content further relates to memory consolidation and retrieval abilities later on.

Finally, it is noteworthy that the relative lack of a cuing effect in the accuracy measures is consistent with the effectiveness of the Shapes task in producing a wakeful rest state. That is, participants reported that they were spending nearly $50 \%$ of their time thinking about the Shapes task, and hence, this is clearly different than a standard intentional learning situation. One would a priori expect that re-presentation of the picture cues or the full picture-word pairs after a delay would significantly boost later memory performance due to the well-established benefits of spacing (Cepeda et al., 2006). A future study that simply re-presents the cues outside of the context of a wakeful rest/Shapes task, but with the same pseudorandom timing intervals used here, would be a useful way to test this hypothesis.

\section{Limitations}

There are a number of important limitations which should be noted. First, one might ask whether the frequency of memory task-related MW reported in these experiments generalizes to real-world scenarios of offline thought and MW. To this point, the average percentage of overall MW (i.e., combined experiment-unrelated MW and memory task-related MW; 25\%) and memory task-related MW ( $\sim 54 \%$ of MW reports) reported across these experiments closely approximates estimates of MW ( 30\%; Kane et al., 2007; Klinger \& Cox, 1987; Killingsworth \& 
Gilbert, 2010) and episodic MW ( $60 \%$ of MW reports; Song \& Wang, 2019) from studies

which have used ecological momentary assessment to estimate thought content frequencies in our everyday lives.

Second, and relatedly, attempting to study these effects in an experimental setting bring about their own unique limitations as well. Specifically, one might be concerned whether there was sufficient time in between the cues and probes presented during the Shapes task. It is also possible that this issue of the time between cues and probes might modulate the results ${ }^{7}$. In addition, it is possible that the targeted reactivation cues might produce a benefit on cued recall in a delayed test (see Roediger \& Karpicke, 2006), which was only minimal in the current immediate testing paradigm. Also, one might question whether testing a wakeful rest study in the context of an online experiment might differ from one run in a controlled laboratory setting, and whether participants were truthful in their responses to post-task questions. Future conceptual replications of this work using different tasks or methodological adjustments will ultimately shed light on these issues.

\section{Conclusions}

The present study revealed several findings regarding the nature of the relationship between MW and episodic memory. First, it appears that MW and episodic memory are linked such that episodic MW may aid in episodic memory consolidation, specifically via trace replay. Second, older adults' reduced MW propensity may contribute to age-related declines in episodic

\footnotetext{
${ }^{7}$ The possibility that the time between the cues and probes presented in the Shapes task influenced thought probe responses and later cueing effects was explored in a set of additional analyses. These analyses yielded the following results: (1) there was no difference in recall accuracy, $t(639.70)=0.15, p=.88$, nor recall accuracy cueing effect, $t(614.70)=1.06, p=.29$, for cues that were closer than the median distance between cues and probes presented during the Shapes task vs. farther than the median distance from probes and (2) there was no difference in correct recall standardized response latencies, $t(509.40)=0.83, p=.41$, nor standardized response latency cueing effect, $t(574.60)=0.59, p=.56$, for cues that were close vs. far from probes. See Appendix E in the Supplemental Materials for additional information.
} 
memory such that younger but not older adults benefited at retrieval from subtle auditory reminder cues. It appears that cues need to be made more salient, and stimulus complexity reduce, for older adults to show a benefit of cues during retrieval. Importantly, there was evidence of a consistent relationship between memory task-related MW and cued episodic memory performance across age groups, experiments, and datasets.

Although the present study certainly informs the aforementioned "scientific blind spot" noted by Gruberger et al. (2011), referencing the dearth of research investigating how MW interacts with other cognitive processes, further work is clearly warranted. Ultimately, the present set of studies suggests that MW may indeed serve the important role of allowing for offline replay of recently encoded information when we are awake but not intentionally encoding new information. This important role of MW may help resolve the tension in the literature regarding the cognitive consequences of the paradoxical result of reduced MW in older adults compared to younger adults (Maillet \& Schacter, 2016). Importantly, the operations that occur as a result of this age-related decline in MW may contribute to age-related deficits in episodic memory retrieval and potentially consolidation as well. 


\section{References}

Alber, J., Della Sala, S., \& Dewar, M. (2014). Minimizing interference with early consolidation boosts 7-day retention in amnesic patients. Neuropsychology, 28(5), 667.

Anderson, N. D., \& Craik, F. I. (2000). Memory in the aging brain. The Oxford handbook of memory, 411-425.

Andrews-Hanna, J. R., Kaiser, R. H., Turner, A. E., Reineberg, A., Godinez, D., Dimidjian, S., \& Banich, M. (2013). A penny for your thoughts: dimensions of self-generated thought content and relationships with individual differences in emotional wellbeing. Frontiers in psychology, 4, 900 .

Andrews-Hanna, J. R., Reidler, J. S., Huang, C., \& Buckner, R. L. (2010). Evidence for the default network's role in spontaneous cognition. Journal of Neurophysiology, 104(1), $322-335$.

Arnau, S., Löffler, C., Rummel, J., Hagemann, D., Wascher, E., \& Schubert, A. L. (2020). Intertrial alpha power indicates mind wandering. Psychophysiology, 57(6), e13581.

Aschenbrenner, A. J., Balota, D. A., Fagan, A. M., Duchek, J. M., Benzinger, T. L., \& Morris, J. C. (2015). Alzheimer disease cerebrospinal fluid biomarkers moderate baseline differences and predict longitudinal change in attentional control and episodic memory composites in the adult children study. Journal of the International Neuropsychological Society, 21(8), 573-583.

Aschenbrenner, A. J., Balota, D. A., Fagan, A. M., Duchek, J. M., Benzinger, T. L., \& Morris, J. C. (2015). Alzheimer disease cerebrospinal fluid biomarkers moderate baseline differences and predict longitudinal change in attentional control and episodic memory 
composites in the adult children study. Journal of the International Neuropsychological Society: JINS, 21(8), 573.

Balota, D. A., Dolan, P. O., \& Duchek, J. M. (2000). Memory changes in healthy young and older adults. The Oxford handbook of memory, 395-410.

Balota, D. A., Yap, M. J., Hutchison, K. A., Cortese, M. J., Kessler, B., Loftis, B., ... \& Treiman, R. (2007). The English lexicon project. Behavior research methods, 39(3), 445-459.

Ben-Yakov, A., Eshel, N., \& Dudai, Y. (2013). Hippocampal immediate poststimulus activity in the encoding of consecutive naturalistic episodes. Journal of Experimental Psychology: General, 142(4), 1255.

Brokaw, K., Tishler, W., Manceor, S., Hamilton, K., Gaulden, A., Parr, E., \& Wamsley, E. J. (2016). Resting state EEG correlates of memory consolidation. Neurobiology of learning and memory, 130, 17-25.

Brysbaert, M., \& New, B. (2009). Moving beyond Kučera and Francis: A critical evaluation of current word frequency norms and the introduction of a new and improved word frequency measure for American English. Behavior research methods, 41(4), 977-990.

Buckner, R. L., Head, D., Parker, J., Fotenos, A. F., Marcus, D., Morris, J. C., \& Snyder, A. Z. (2004). A unified approach for morphometric and functional data analysis in young, old, and demented adults using automated atlas-based head size normalization: Reliability and validation against manual measurement of total intracranial volume. NeuroImage, 23, $724-738$.

Buzsáki, G. (1989). A two-stage model of memory trace formation: A role for "noisy" brain states. Neuroscience, $31,551-570$. 
Callard, F., Smallwood, J., Golchert, J., \& Margulies, D. S. (2013). The era of the wandering mind? Twenty-first century research on self-generated mental activity. Frontiers in psychology, 4, 891 .

Cepeda, N. J., Vul, E., Rohrer, D., Wixted, J. T., \& Pashler, H. (2008). Spacing effects in learning: A temporal ridgeline of optimal retention. Psychological science, 19(11), 10951102.

Christoff, K., Gordon, A., \& Smith, R. (2011). The role of spontaneous thought in human cognition. In Neuroscience of decision making (pp. 271-296). Psychology Press.

Cohen, J. (1988). Statistical power analysis for the behavioral sciences (2nd ed.). Hillsdale, NJ: Erlbaum.

Craig, M., \& Dewar, M. (2018). Rest-related consolidation protects the fine detail of new memories. Scientific reports, 8(1), 1-9.

Craig, M., Della Sala, S., \& Dewar, M. (2014). Autobiographical thinking interferes with episodic memory consolidation. PloS one, 9(4).

Craig, M., Dewar, M., Della Sala, S., \& Wolbers, T. (2015). Rest boosts the long-term retention of spatial associative and temporal order information. Hippocampus, 25(9), 1017-1027.

Craig, M., Dewar, M., Harris, M. A., Della Sala, S., \& Wolbers, T. (2016). Wakeful rest promotes the integration of spatial memories into accurate cognitive maps. Hippocampus, 26(2), 185-193.

Craig, M., Ottaway, G., \& Dewar, M. (2018). Rest on it: Awake quiescence facilitates insight. Cortex, 109, 205-214. 
Desikan, R. S., Ségonne, F., Fischl, B., Quinn, B. T., Dickerson, B. C., Blacker, D., \& Killiany, R. J. (2006). An automated labeling system for subdividing the human cerebral cortex on MRI scans into gyral based regions of interest. NeuroImage, 31, 968-980.

Dewar, M. T., \& Cowan, N. (2010). Forgetting due to retroactive interference in amnesia: Findings and implications In S. Della Sala (Ed.), Forgetting (pp. 185-209).

Dewar, M., Alber, J., Butler, C., Cowan, N., \& Della Sala, S. (2012). Brief wakeful resting boosts new memories over the long term. Psychological science, 23(9), 955-960.

Dewar, M., Alber, J., Cowan, N., \& Della Sala, S. (2014). Boosting long-term memory via wakeful rest: intentional rehearsal is not necessary, consolidation is sufficient. PloS one, $9(10), \mathrm{e} 109542$.

Dewar, M., Garcia, Y. F., Cowan, N., \& Sala, S. D. (2009). Delaying interference enhances memory consolidation in amnesic patients. Neuropsychology, 23(5), 627.

Duchek, J. M. (1984). Encoding and retrieval differences between young and old: The impact of attentional capacity usage. Developmental Psychology, 20(6), 1173.

Ellenbogen, J. M., Payne, J. D., \& Stickgold, R. (2006). The role of sleep in declarative memory consolidation: passive, permissive, active or none? Current opinion in neurobiology, $16(6), 716-722$

Faust, M. E., Balota, D. A., Spieler, D. H., \& Ferraro, F. R. (1999). Individual differences in information-processing rate and amount: implications for group differences in response latency. Psychological bulletin, 125(6), 777.

Fischer, S., \& Born, J. (2009). Anticipated reward enhances offline learning during sleep. Journal of Experimental Psychology: Learning, Memory, and Cognition, 35(6), 1586. 
Fischl, B. A., van der Kouwe, A., Destrieux, C., Halgren, E., Segonne, F., Salat, D., \& Dale, A. M. (2004). Automatically parcellating the human cerebral cortex. Cerebral Cortex, 14, 11-22.

Fischl, B., Salat, D. H., Busa, E., Albert, M., Dieterich, M., Haselgrove, C., \& Dale, A. M. (2002). Whole brain segmentation: Automated labeling of neuroanatomical structures in the human brain. Neuron, 33, 341-355.

Frank, D. J., Nara, B., Zavagnin, M., Touron, D. R., \& Kane, M. J. (2015). Validating older adults' reports of less mind-wandering: An examination of eye movements and dispositional influences. Psychology and Aging, 30(2), 266.

Giambra, L. M. (1979). Sex differences in daydreaming and related mental activity from the late teens to the early nineties. Int. J. Aging Hum. Dev. 10, 1-34.

Giambra, L. M., and Grodsky, A. (1989). Task-unrelated images and thoughts while reading. Imagery Curr. Perspect. 26-31.

Gilbert, S. J., Frith, C. D., and Burgess, P. W. (2005). Involvement of rostral prefrontal cortex in selection between stimulus-oriented and stimulus-independent thought. Eur. J. Neurosci. $21,1423-1431$.

Gottselig, J., Hofer-Tinguely, G., Borbely, A., Regel, S., Landolt, H., Retey, J., \& Achermann, P. (2004). Sleep and rest facilitate auditory learning. Neuroscience, 127(3), 557-561.

Grober, E., Buschke, H. C. H. B. S., Crystal, H., Bang, S., \& Dresner, R. (1988). Screening for dementia by memory testing. Neurology, 38(6), 900-900.

Gruberger, M., Simon, E. B., Levkovitz, Y., Zangen, A., \& Hendler, T. (2011). Towards a neuroscience of mind-wandering. Frontiers in human neuroscience, 5, 56. 
Hasselmo, M. (1999). Neuromodulation: Acetylcholine and memory consolidation. Trends in Cognitive Science, 3, 351-359.

Head, D., Rodrigue, K. M., \& Kennedy, K. M. (2008). Neuroanatomical and cognitive mediators of age-related differences in episodic memory. Neuropsychology, 22, 491-507.

Hu, P., Stylos-Allan, M., \& Walker, M. P. (2006). Sleep facilitates consolidation of emotional declarative memory. Psychological science, 17(10), 891-898.

Humiston, G., \& Wamsley, E. (2018). A breif period of eyes-closed rest enhances motor skill consolidation. Neurobiology of Learning and Memory, 155, 1-6.

Jack, C. R. J., Twomey, C. K., Zinsmeister, A. R., Sharbrough, F. W., Petersen, R. C., \& Cascino, G. D.(1989).Anterior temporallobes andhippocampalformations:Normative volumetric measurements from MR images in young adults. Radiology, 172, 549-554.

Jackson, J. D., \& Balota, D. A. (2012). Mind-wandering in younger and older adults: Converging evidence from the sustained attention to response task and reading for comprehension. Psychology and aging, 27(1), 106.

Jadhav, S. P., Kemere, C., German, P. W., \& Frank, L. M. (2012). Awake hippocampal sharpwave ripples support spatial memory. Science, 336(6087), 1454-1458.

James, W. (1892). Text-book of Psychology. Macmillan.

Kane, M. J., Brown, L. H., McVay, J. C., Silvia, P. J., Myin-Germeys, I., \& Kwapil, T. R. (2007). For whom the mind wanders, and when: An experience-sampling study of working memory and executive control in daily life. Psychological science, 18(7), 614621.

Kane, M. J., Brown, L. H., McVay, J. C., Silvia, P. J., Myin-Germeys, I., \& Kwapil, T. R. (2007). For whom the mind wanders, and when: An experience-sampling study of 
working memory and executive control in daily life. Psychological science, 18(7), 614621.

Karlsson, M. and Frank, L. (2009) Awake replay of remote experiences in the hippocampus. Nature Neuroscience, 12, 913-918.

Killingsworth, M. A., \& Gilbert, D. T. (2010). A wandering mind is an unhappy mind. Science, $330(6006), 932-932$.

Klinger, E., \& Cox, W. M. (1987). Dimensions of thought flow in everyday life. Imagination, Cognition and Personality, 7(2), 105-128.

Klinzing, J. G., Niethard, N., \& Born, J. (2019). Mechanisms of systems memory consolidation during sleep. Nature neuroscience, 22(10), 1598-1610.

Koen, J. D., \& Yonelinas, A. P. (2014). The effects of healthy aging, amnestic mild cognitive impairment, and Alzheimer's disease on recollection and familiarity: A meta-analytic review. Neuropsychology review, 24(3), 332-354.

Krawietz, S. A., Tamplin, A. K., \& Radvansky, G. A. (2012). Aging and mind wandering during text comprehension. Psychology and aging, 27(4), 951.

Lansink, C. S., Goltstein, P. M., Lankelma, J. V., Joosten, R. N., McNaughton, B. L., \& Pennartz, C. M. (2008). Preferential reactivation of motivationally relevant information in the ventral striatum. Journal of Neuroscience, 28(25), 6372-6382.

Lansink, C. S., Goltstein, P. M., Lankelma, J. V., McNaughton, B. L., \& Pennartz, C. M. (2009). Hippocampus leads ventral striatum in replay of place-reward information. PLoS Biol, 7(8), e1000173. 
Lau, H., Tucker, M. A., \& Fishbein, W. (2010). Daytime napping: Effects on human direct associative and relational memory. Neurobiology of learning and memory, 93(4), 554560.

Maillet, D., \& Schacter, D. L. (2016). From mind wandering to involuntary retrieval: Age-related differences in spontaneous cognitive processes. Neuropsychologia, 80, 142-156.

Marr, D. (1971). Simple memory: A theory for archicortex. Philosophical Transactions of the Royal Society of London, Series B, Biological Sciences, 262, 23-81.

Mathalon, D. H., Sullivan, E. V., Rawles, J. M., \& Pfefferbaum, A. (1993). Correction for head size in brain-imaging measurements. Psychiatry Research: Neuroimaging, 50, 121-139

Mednick, S., Makovski, T., Cai, D., \& Jiang, Y. (2009). Hippocampal-dependent implicit visual memory improves with practice, not sleep. Journal of Vision, 9(8), 572-572.

Mercer, T. (2015). Wakeful rest alleviates interference-based forgetting. Memory, 23(2), 127137.

Mildner, J. N., \& Tamir, D. I. (2019). Spontaneous thought as an unconstrained memory process. Trends in neurosciences, 42(11), 763-777.

Millar, P. R., \& Balota, D. A. (2018). Wakeful rest benefits memory when materials can be rehearsed. BioRxiv, 449835.

Millar, P. R., Balota, D. A., Maddox, G. B., Duchek, J. M., Aschenbrenner, A. J., Fagan, A. M., ... \& Morris, J. C. (2017). Process dissociation analyses of memory changes in healthy aging, preclinical, and very mild Alzheimer disease: Evidence for isolated recollection deficits. Neuropsychology, 31(7), 708.

Mills, C., Herrera-Bennett, A., Faber, M., \& Christoff, K. (2018). Why the mind wanders: How spontaneous thought's default variability may support episodic efficiency and semantic 
optimization. The Oxford handbook of spontaneous thought: mind-wandering, creativity, and dreaming, 11-22.

Morin, A. (2009). Self-awareness deficits following loss of inner speech: Dr. Jill Bolte Taylor's case study. Consciousness and cognition, 18(2), 524-529.

Müller, G., \& Pizecker, A. (1900). Experimentelle Beiträge zur Lehre vom Gedächtnis. Z. Psychol. Ergänzungsband (Experimental contributions to the science of memory), 1, 1300.

Naveh-Benjamin, M. (2000). Adult age differences in memory performance: tests of an associative deficit hypothesis. Journal of Experimental Psychology: Learning, Memory, and Cognition, 26(5), 1170.

Naveh-Benjamin, M., Guez, J., \& Shulman, S. (2004). Older adults' associative deficit in episodic memory: Assessing the role of decline in attentional resources. Psychonomic Bulletin \& Review, 11(6), 1067-1073.

Nicosia, J. \& Balota, D. (2021). Disppositional Factors Fully Account for Age-Differences in Mind-Wandering. Psychology and Aging, 36(4), 421-432.

O'Callaghan, C., Walpola, I. C., \& Shine, J. M. (2021). Neuromodulation of the mind-wandering brain state: the interaction between neuromodulatory tone, sharp wave-ripples and spontaneous thought. Philosophical Transactions of the Royal Society B, 376(1817), 20190699.

O’Callaghan, C., Shine, J. M., Lewis, S. J., Andrews-Hanna, J. R., \& Irish, M. (2015). Shaped by our thoughts-A new task to assess spontaneous cognition and its associated neural correlates in the default network. Brain and cognition, 93, 1-10. 
O’Keefe, J. \& Distrovsky, J. (1971). The hippocampus as a spatial map: Preliminary evidence from unit activity in the freely-moving rat. Brain Research, 34, 1717-175.

Olejnik, S., \& Algina, J. (2003). Generalized eta and omega squared statistics: measures of effect size for some common research designs. Psychological methods, 8(4), 434.

Oudiette, D., Antony, J. W., Creery, J. D., \& Paller, K. A. (2013). The role of memory reactivation during wakefulness and sleep in determining which memories endure. Journal of Neuroscience, 33(15), 6672-6678.

Peigneux, P., Laureys, S., Fuchs, S., Collette, F., Perrin, F., et al. (2004). Are spatial memories strengthened in the human hippocampus during slow wave sleep? Neuron, 44, 535 -545.

Peigneux, P., Laureys, S., Fuchs, S., Collette, F., Perrin, F., et al. (2004). Are spatial memories strengthened in the human hippocampus during slow wave sleep? Neuron, 44, 535-545.

Peirce, J., Gray, J. R., Simpson, S., MacAskill, M., Höchenberger, R., Sogo, H., ... \& Lindeløv, J. K. (2019). PsychoPy2: Experiments in behavior made easy. Behavior research methods, 51(1), 195-203.

Rabinowitz, J. C., Craik, F. I., \& Ackerman, B. P. (1982). A processing resource account of age differences in recall. Canadian Journal of Psychology/Revue canadienne de psychologie, $36(2), 325$.

Rasch, B., Born, J., \& Gais, S. (2006). Combined blockade of cholinergic receptors shifts the brain from stimulus encoding to memory consolidation. Journal of Cognitive Neuroscience, $18,793-802$.

Rasch, B., Buchel, C., Gais, S., \& Born, J. (2007). Odor cues during slow-wave sleep prompt declarative memory consolidation. Science, 315, 1426-1429. 
Raz, N., Lindenberger, U., Ghisletta, P., Rodrigue, K. M., Kennedy, K. M., \& Acker, J. D. (2008). Neuroanatomical correlates of fluid intelligence in healthy adults and persons with vascular risk factors. Cerebral Cortex, 18, 718-726.

Roediger III, H. L., \& Karpicke, J. D. (2006). Test-enhanced learning: Taking memory tests improves long-term retention. Psychological science, 17(3), 249-255.

Salat, D. H., Greve, D. N., Pacheco, J. L., Quinn, B. T., Helmer, K. G., Buckner, R. L., \& Fischl, B. (2009). Regional white matter volume differences in nondemented aging and Alzheimer's disease. NeuroImage, 44, 1247-1258.

Singer, J. L., \& McCraven, V. G. (1961). Some characteristics of adult daydreaming. The Journal of psychology, 51(1), 151-164.

Sirota, A., Csicsvari, J. Buhl, D., \& Buzsáki, G. (2003). Communication between neocortex and hippocampus during sleep in rats and mice. Proceedings of the National Academy of Sciences, 100, 206-2069.

Smallwood, J., \& Schooler, J. W. (2015). The science of mind wandering: empirically navigating the stream of consciousness. Annual review of psychology, 66, 487-518.

Smallwood, J., Fishman, D. J., \& Schooler, J. W. (2007). Counting the cost of an absent mind: Mind wandering as an underrecognized influence on educational performance. Psychonomic bulletin \& review, 14(2), 230-236.

Smits, C., Kapteyn, T. S., \& Houtgast, T. (2004). Development and validation of an automatic speech-in-noise screening test by telephone. International journal of audiology, 43(1), $15-28$.

Song, X., \& Wang, X. (2012). Mind wandering in Chinese daily lives-an experience sampling study. PloS one, 7(9). 
Sterpenich, V., Albouy, G., Darsaud, A., Schmidt, C., Vandewalle, G., Vu, T. T. D., ... \& Maquet, P. (2009). Sleep promotes the neural reorganization of remote emotional memory. Journal of Neuroscience, 29(16), 5143-5152.

Szpunar, K. K., Watson, J. M., \& McDermott, K. B. (2007). Neural substrates of envisioning the future. Proceedings of the National Academy of Sciences, 104(2), 642-647.

Takashima, A., Nieuwenhuis, I., Jensen, O., Talamini, L., Rijpkema, M., \& Fernández, G. (2009). Shift from hippocampal to neocortical centered retrieval network with consolidation. Journal of Neuroscience, 29, $10087-10093$.

Tambini, A., Ketz, N., \& Davachi, L. (2010). Enhanced brain correlations during rest are related to memory for recent experiences. Neuron, 65(2), 280-290.

Teasdale, J. D., Dritschel, B. H., Taylor, M. J., Proctor, L., Lloyd, C. A., Nimmo-Smith, I., \& Baddeley, A. D. (1995). Stimulus-independent thought depends on central executive resources. Memory \& cognition, 23(5), 551-559.

Tononi, G., \& Cirelli, C. (2006). Sleep function and synaptic homeostasis. Sleep medicine reviews, 10(1), 49-62.

Van Dongen, E. V., Thielen, J. W., Takashima, A., Barth, M., \& Fernández, G. (2012). Sleep supports selective retention of associative memories based on relevance for future utilization. PloS one, 7(8), e43426.

Varma, S., Daselaar, S. M., Kessels, R. P., \& Takashima, A. (2018). Promotion and suppression of autobiographical thinking differentially affect episodic memory consolidation. PloS one, 13(8), e0201780. 
Varma, S., Takashima, A., Krewinkel, S., Van Kooten, M., Fu, L., Medendorp, W. P., ... \& Daselaar, S. M. (2017). Non-interfering effects of active post-encoding tasks on episodic memory consolidation in humans. Frontiers in behavioral neuroscience, 11, 54.

Varma, S., Takashima, A., Fu, L., \& Kessels, R. P. (2019). Mindwandering propensity modulates episodic memory consolidation. Aging clinical and experimental research, 31(11), 16011607.

Wahlheim, C. N., \& Jacoby, L. L. (2013). Remembering change: The critical role of recursive remindings in proactive effects of memory. Memory \& Cognition, 41(1), 1-15.

Wamsley, E. J. (2019). Memory consolidation during waking rest. Trends in cognitive sciences, 23(3), 171-173.

Wang, K., Yu, C., Xu, L., Qin, W., Li, K., Xu, L., \& Jiang, T. (2009). Offline memory reprocessing: involvement of the brain's default network in spontaneous thought processes. PLoS One, 4(3).

Wechsler, D. Manual: Wechsler Memory Scale-Revised. Psychological Corporation; San Antonio, Texas: 1987.

Wechsler, D.; Stone, CP. Manual: Wechsler Memory Scale. Psychological Corporation; New York: 1973.

Wilhelm, I., Diekelmann, S., Molzow, I., Ayoub, A., Mölle, M., \& Born, J. (2011). Sleep selectively enhances memory expected to be of future relevance. Journal of Neuroscience, 31(5), 1563-1569.

Wilson, M. A., \& McNaughton, B. L. (1994). Reactivation of hippocampal ensemble memories during sleep. Science, 265, 676-679. 
Wixted, J. (2004). The psychology and neuroscience of forgetting. Annual Review of Psychology, 55, 235-269.

Wixted, J. T., \& Cai, D. J. (2014). Memory consolidation. In K. N. Ochsner \& S. M. Kosslyn (Eds.), Oxford library of psychology. The Oxford handbook of cognitive neuroscience, Vol. 1. Core topics (p. 436-455). Oxford University Press.

Zacks, R. T., \& Hasher, L. (1994). Directed ignoring: Inhibitory regulation of working memory. 
Tables \& Figures

\begin{tabular}{lllcccc}
\hline Experiment & Age Group & $\mathbf{N}$ & Age (Years) [Range] & WMS Acc. (\%) & $\boldsymbol{t}$ & $\boldsymbol{p}$ \\
\hline \multirow{2}{*}{ Experiment 1 } & Younger & 60 & $22.72[18-30]$ & 74.83 & \multirow{2}{*}{1.76} & \multirow{2}{*}{.08} \\
& Older & 58 & $69.75[62-89]$ & 67.46 & & \\
\hline \multirow{2}{*}{ Experiment 2 } & Younger & 55 & $23.85[18-31]$ & 82.73 & \multirow{2}{*}{4.24} & $<.001$ \\
& Older & 60 & $68.66[62-80]$ & 68.20 & & \\
\hline \multirow{2}{*}{ Experiment 3 } & Younger & 54 & $22.70[18-31]$ & 84.44 & \multirow{2}{*}{4.71} & $<.001$ \\
& Older & 56 & $68.59[62-83]$ & 69.14 & & \\
\hline
\end{tabular}

Table 1. Demographic information. 


\begin{tabular}{llcccc}
\hline Experiment & Probe Response & Younger & Older & $\boldsymbol{t}$ & $\boldsymbol{p}$ \\
\hline \multirow{3}{*}{ Experiment 1 } & On Task & 49.30 & 56.30 & 1.60 & .11 \\
& Memory MW & 28.99 & 32.46 & 0.91 & .37 \\
& MW & 21.71 & 11.24 & 3.19 & .002 \\
\hline \multirow{5}{*}{ Experiment 2 } & On Task & 35.83 & 55.03 & 4.65 & $<.001$ \\
& MW & 45.30 & 32.08 & 3.26 & .001 \\
Experiment 3 & Memory MW & 28.43 & 30.33 & 0.53 & .60 \\
& MW & 30.06 & 12.37 & 3.39 & $<.001$ \\
\hline
\end{tabular}

Table 2. Thought probe response percentages as a function of Age Group and Experiment. 
Encoding Task

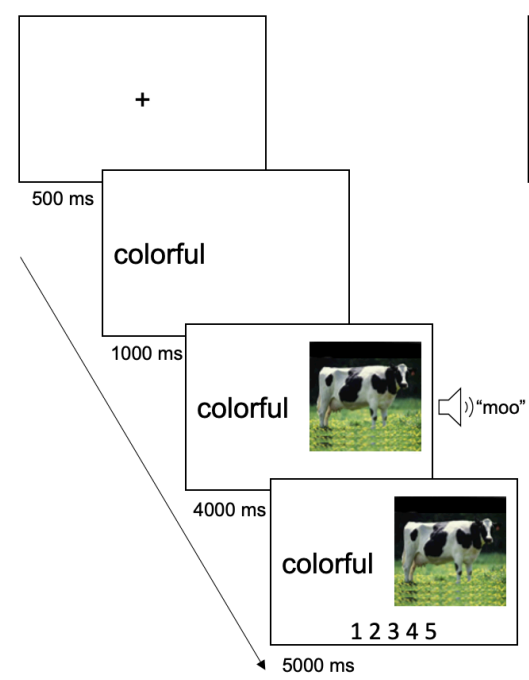

Shapes Task

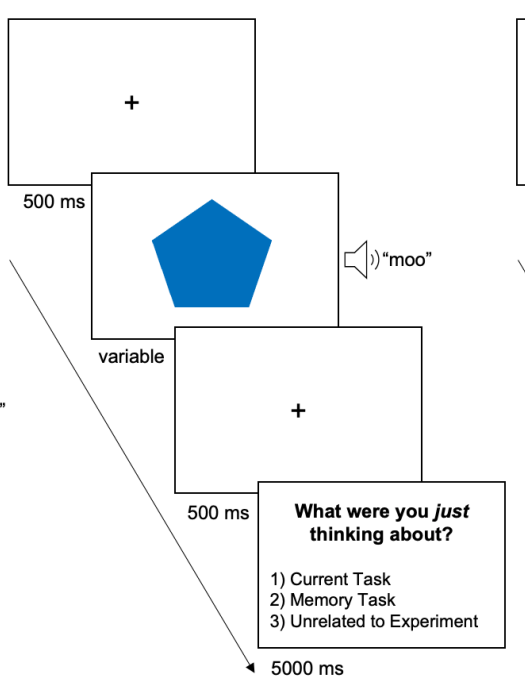

Recall Task

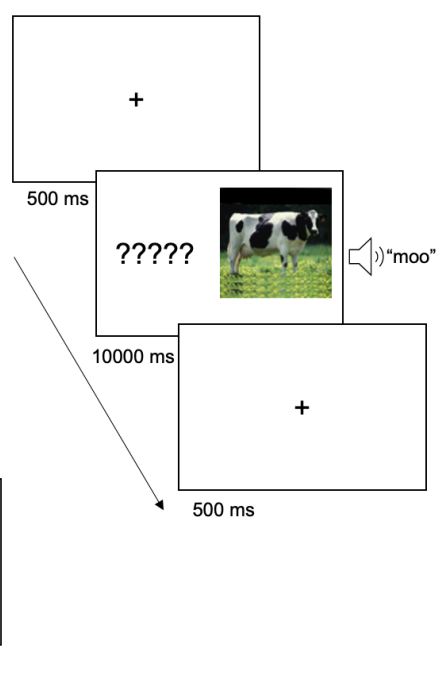

Figure 1. General experimental design. Variable interval in the Shapes task was 3-42 seconds. 


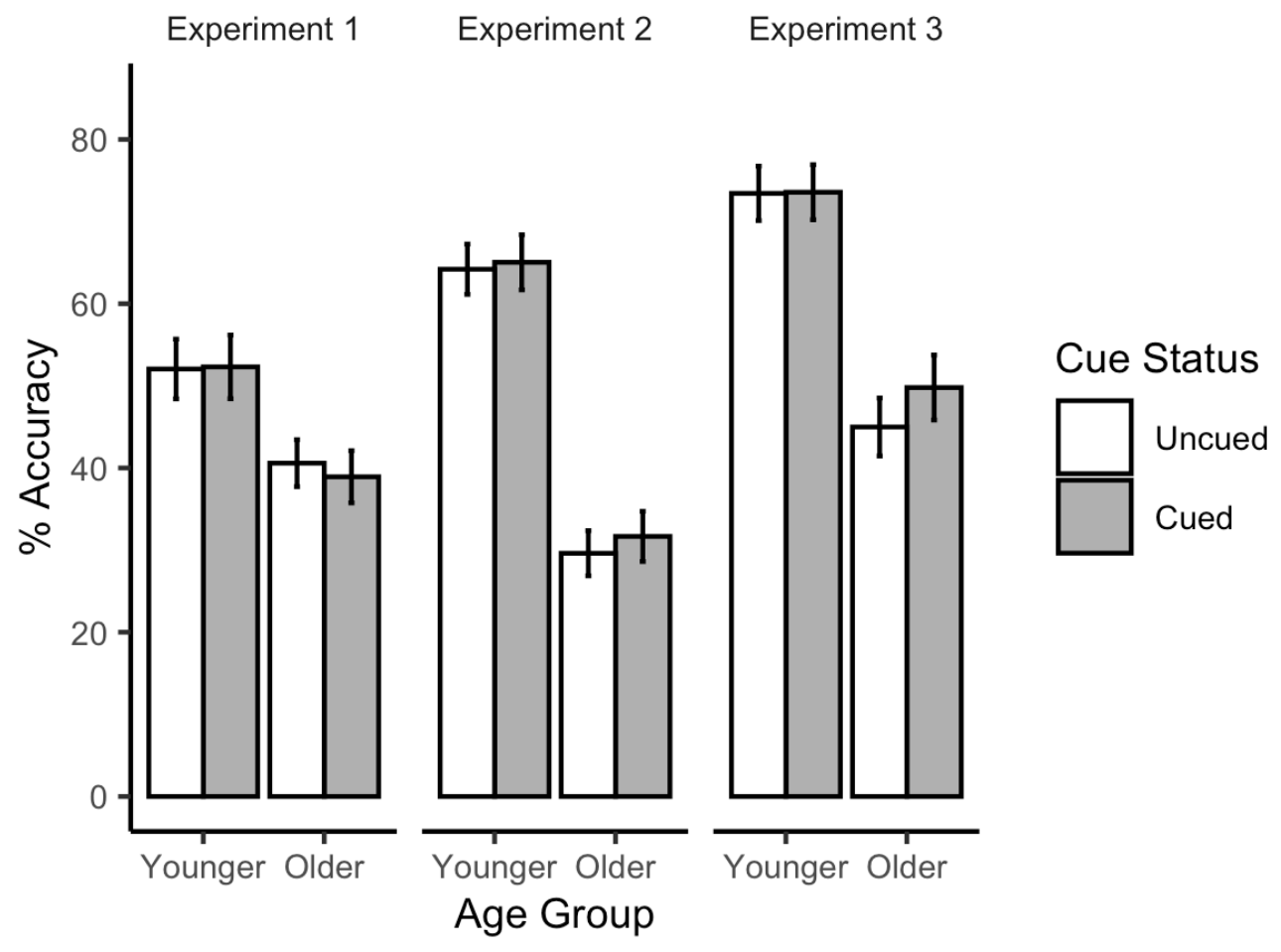

Figure 2. Recall accuracy as a function of Age Group (Younger, Older) and Cue Status (Uncued, Cued) for Experiments1, 2, and 3. Error bars represent standard error of the mean. 


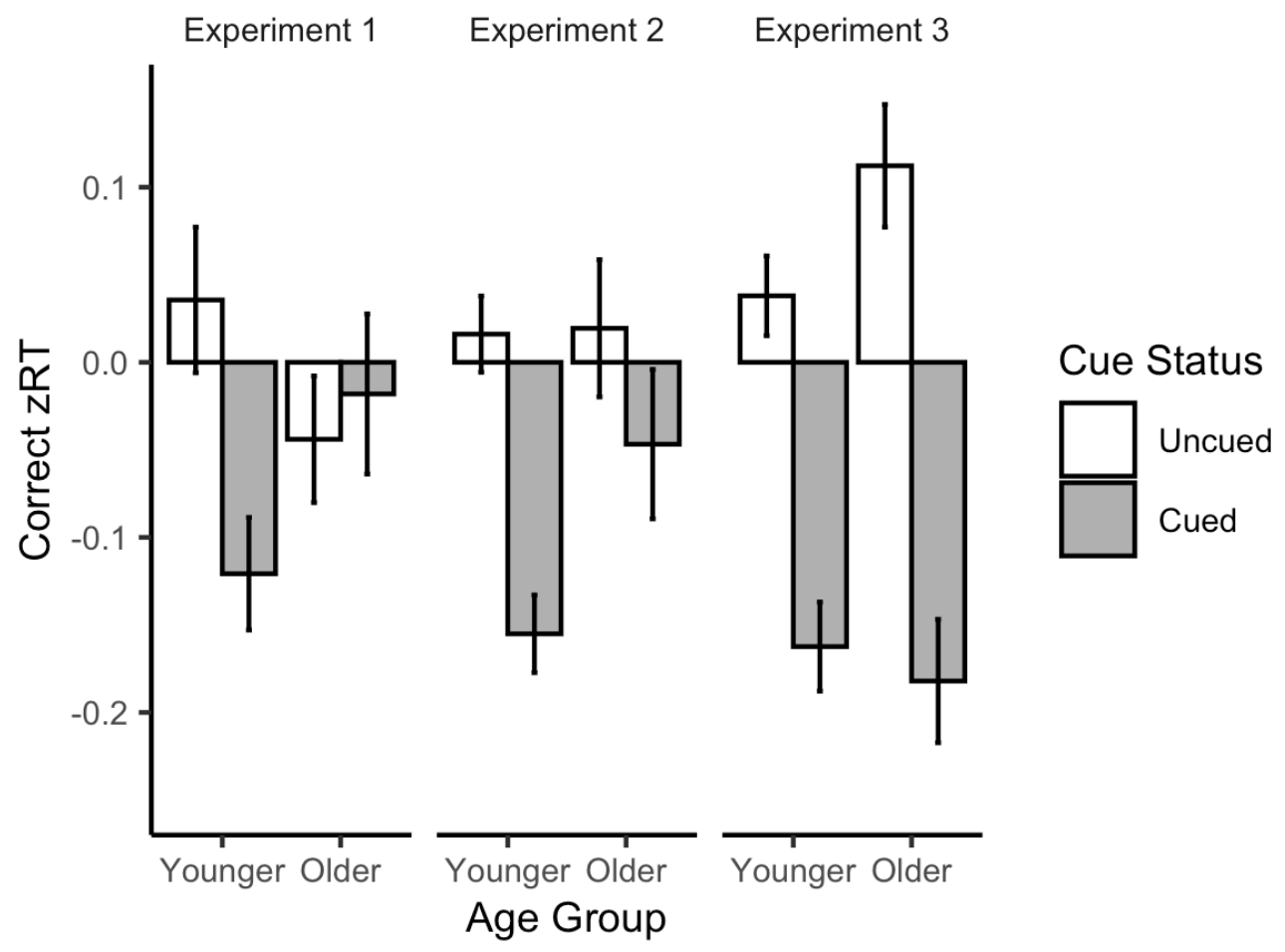

Figure 3. Correct recall standardized response times as a function of Age Group (Younger,

Older) and Cue Status (Uncued, Cued) for Experiments 1, 2, and 3. Error bars represent standard error of the mean. 

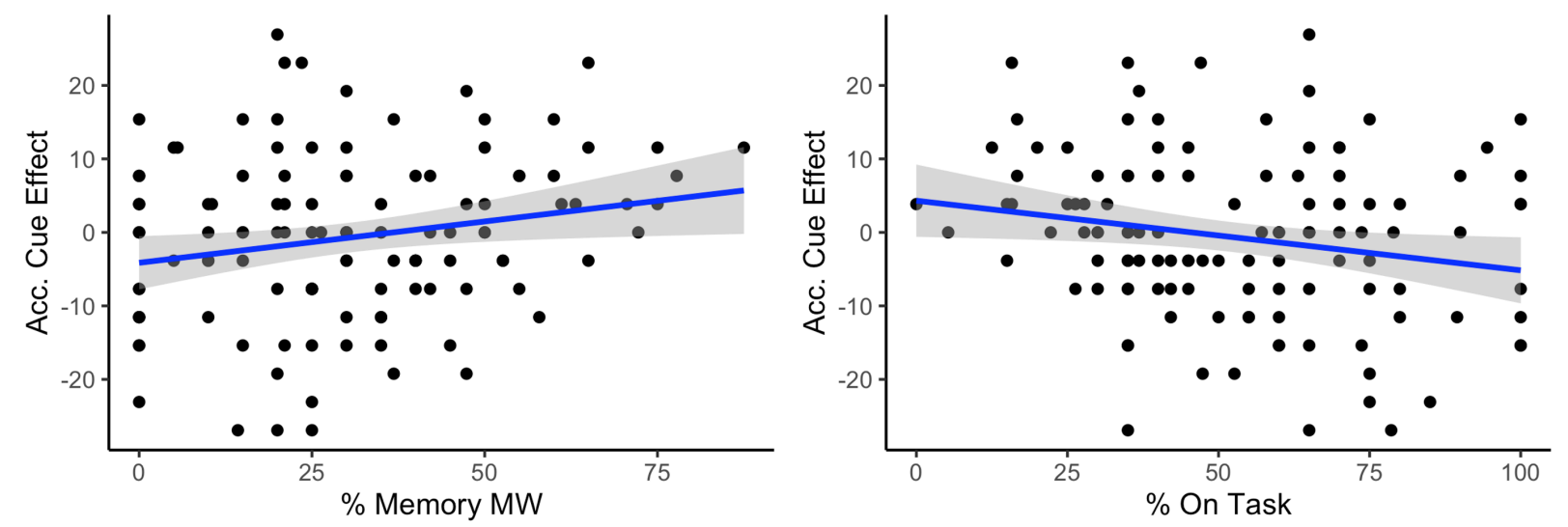

Figure 4. Scatterplots showing the relationship between thought probe response percentages and the accuracy cueing effect for Experiment 1. 

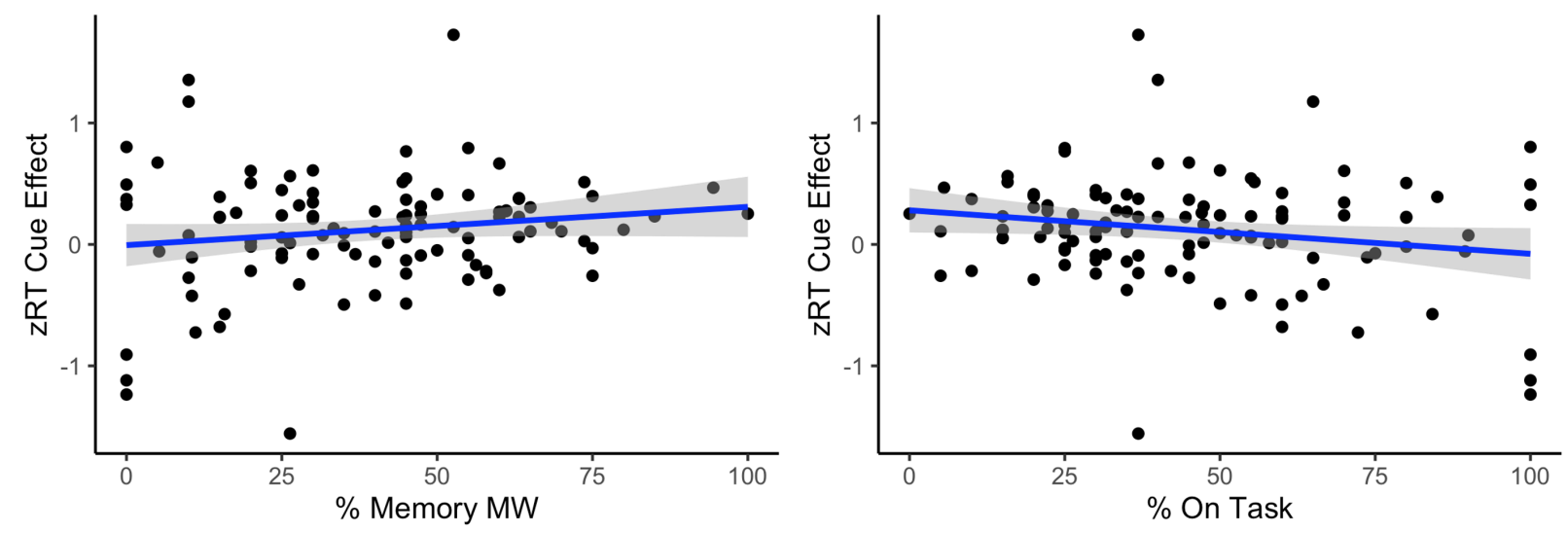

Figure 5. Scatterplots showing the relationship between thought probe response percentages and the zRT cueing effect for Experiment 2. 


\section{Supplemental Materials}

Appendix A

\begin{tabular}{lllccc}
\hline Experiment & Age Group & N & Retrieval During Shapes & Distractions & Wrote Down \\
\hline \multirow{2}{*}{ Experiment 1 } & Younger & 60 & 30 & 5 & 0 \\
& Older & 58 & 33 & 1 & 4 \\
\hline \multirow{2}{*}{ Experiment 2 } & Younger & 55 & 50 & 8 & 7 \\
& Older & 60 & 51 & 1 & 6 \\
\hline \multirow{2}{*}{ Experiment 3 } & Younger & 54 & 43 & 11 & 6 \\
& Older & 56 & 46 & 3 & 5 \\
\hline
\end{tabular}

Post-task questionnaire data as a function of Experiment and Age Group. Analyses of Experiment 1 data split by participants' reports to whether they tried to retrieve the encoding information during the Shapes task indicated that this did not change any of the reported findings. Participants who reported that they wrote things down during the experiment were removed and replaced before analysis. 
Appendix B

\begin{tabular}{lllcccc}
\hline Experiment & Age Group & N & Hearing Aid & Subtitles & Conversation Difficulty & Experiment Difficulty \\
\hline \multirow{2}{*}{ Experiment 1 } & Younger & 60 & --- & --- & --- & --- \\
& Older & 58 & 0 & 2 & 1.39 & 4.02 \\
\hline \multirow{2}{*}{ Experiment 2 } & Younger & 55 & --- & --- & --- & --- \\
& Older & 60 & 1 & 0 & 1.56 & 3.73 \\
\hline \multirow{2}{*}{ Experiment 3 } & Younger & 60 & --- & --- & --- & --- \\
& Older & 60 & --- & --- & --- & -- \\
\hline
\end{tabular}

Hearing questionnaire data as a function of Experiment and Age Group. Removal of participants who reported using hearing aids or subtitles did not change any of the reported findings. 
Appendix C

\begin{tabular}{lllccc}
\hline Condition & Age Group & N & Uncued RT & Cued RT & RT Cue Effect \\
\hline \multirow{2}{*}{ Experiment 1 } & Younger & 55 & 2515 & 2355 & 160 \\
& Older & 60 & 3080 & 3071 & 9 \\
\hline \multirow{2}{*}{ Experiment 2 } & Younger & 60 & 2644 & 2342 & 302 \\
& Older & 58 & 3334 & 3363 & -29 \\
\hline \multirow{2}{*}{ Experiment 3 } & Younger & 54 & 2366 & 2149 & 217 \\
& Older & 56 & 3033 & 2551 & 482 \\
\hline
\end{tabular}

Correct recall RT as a function of Age Group, Cue Status, and Experiment. RT Cue Effect is calculated by subtracting the Cued RT from the Uncued RT $($ RT Cue Effect $=$ Uncued RT - Cued RT). 


\begin{tabular}{|c|c|c|c|c|c|c|}
\hline & \multicolumn{6}{|c|}{ Appendix D } \\
\hline Variable & 1 & 2 & 3 & 4 & 5 & 6 \\
\hline 1. \% On Task & & & & & & \\
\hline 2. \% Memory Task MW & $\begin{array}{c}-.64 * * \\
{[-.70,-.57]}\end{array}$ & & & & & \\
\hline 3. \% MW & $\begin{array}{c}-.57 * * \\
{[-.64,-.49]}\end{array}$ & $\begin{array}{c}-.27 * * \\
{[-.36,-.17]}\end{array}$ & & & & \\
\hline 4. Accuracy Cue Effect & $\begin{array}{c}-.09 \\
{[-.20, .01]}\end{array}$ & $\begin{array}{c}.11 * \\
{[.01, .22]}\end{array}$ & $\begin{array}{c}-.01 \\
{[-.11, .10]}\end{array}$ & & & \\
\hline 5. zRT Cue Effect & $\begin{array}{c}-.06 \\
{[-.17, .04]}\end{array}$ & $\begin{array}{c}.09 \\
{[-.02, .20]}\end{array}$ & $\begin{array}{c}-.02 \\
{[-.12, .09]}\end{array}$ & $\begin{array}{c}.07 \\
{[-.04, .18]}\end{array}$ & & \\
\hline 6. \% Total Accuracy & $\begin{array}{c}-.19 * * \\
{[-.29,-.09]}\end{array}$ & $\begin{array}{c}.15^{* *} \\
{[.04, .25]}\end{array}$ & $\begin{array}{c}.08 \\
{[-.02, .19]}\end{array}$ & $\begin{array}{c}.15 * * \\
{[.04, .25]}\end{array}$ & $\begin{array}{c}.05 \\
{[-.06, .16]}\end{array}$ & \\
\hline 7. \% WMS Accuracy & $\begin{array}{c}-.10 \\
{[-.20, .01]}\end{array}$ & $\begin{array}{c}.12 * \\
{[.01, .22]}\end{array}$ & $\begin{array}{c}-.01 \\
{[-.11, .10]}\end{array}$ & $\begin{array}{c}.01 \\
{[-.09, .12]}\end{array}$ & $\begin{array}{c}.05 \\
{[-.06, .16]}\end{array}$ & $\begin{array}{c}.54 * * \\
{[.46, .61]}\end{array}$ \\
\hline
\end{tabular}

Zero-order correlations between thought probe responses, cue effects, total cued recall accuracy, and WMS accuracy collapsing across Experiments 1, 2, and 3. Values in brackets represent the $95 \%$ confidence interval. * indicates $\mathrm{p}<.05$ and $* *$ indicates $\mathrm{p}<.01$. 


\section{Appendix E}

(1)

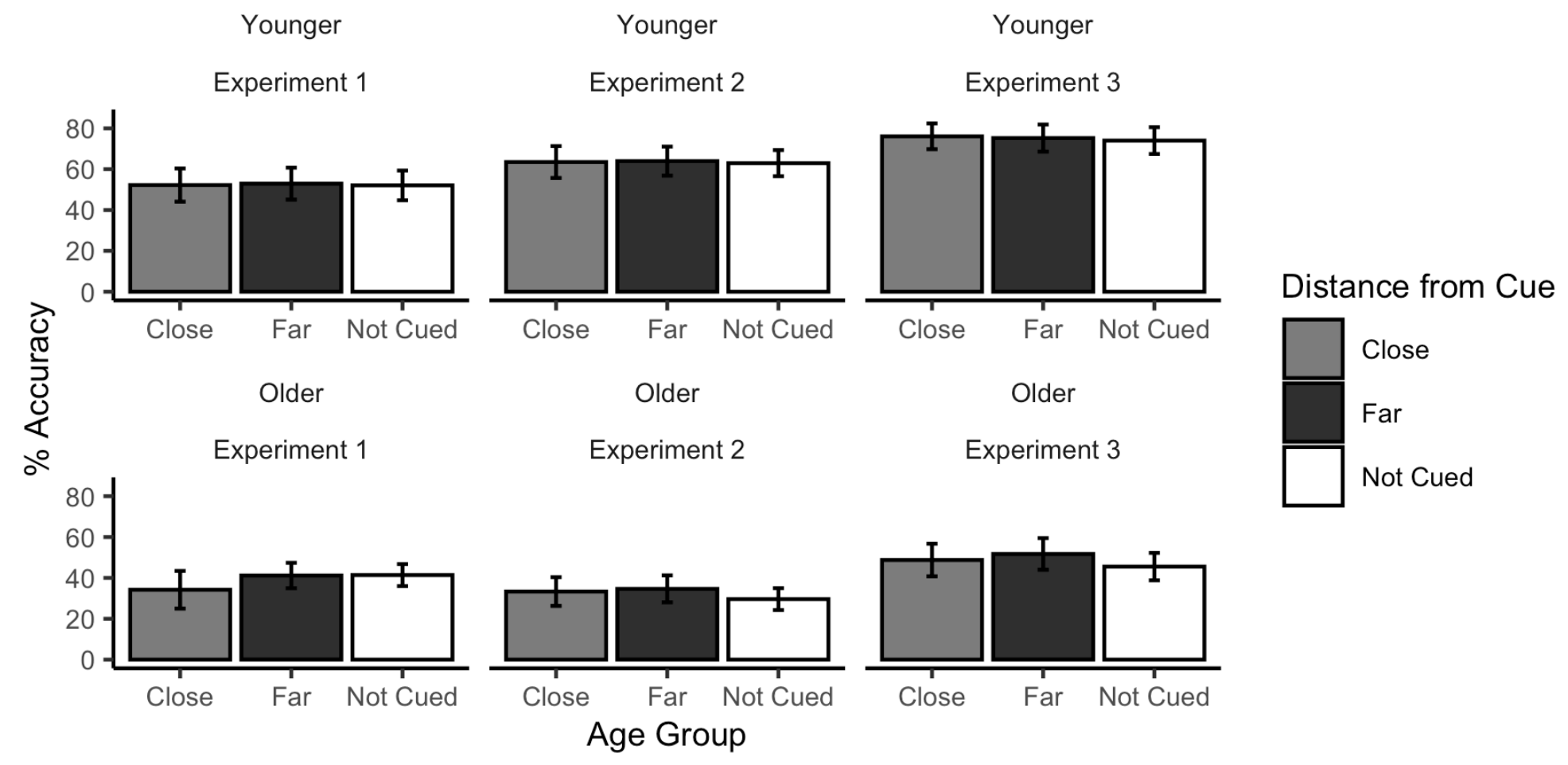


(2)

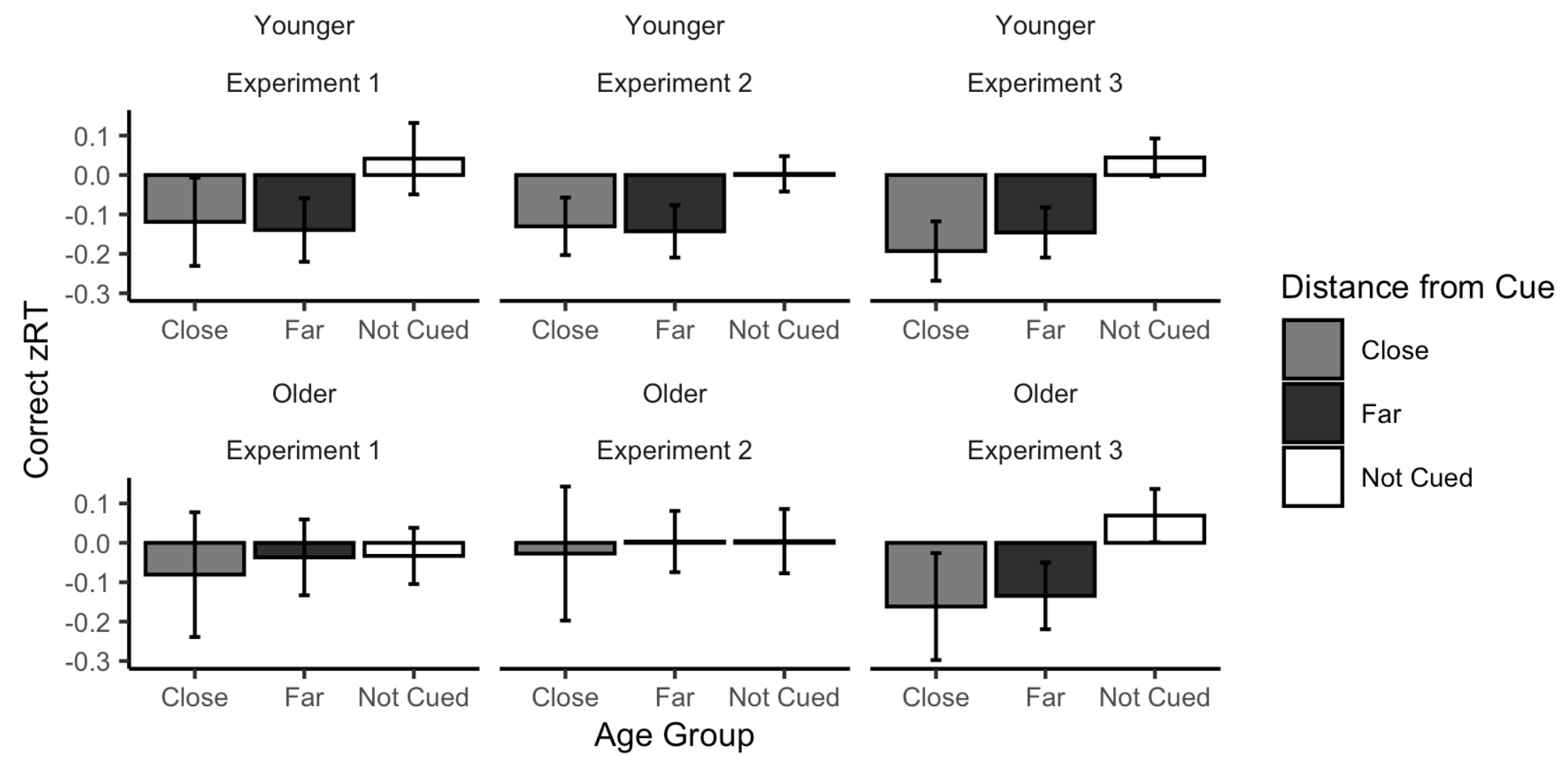

The possibility that the time between the cues and probes presented in the Shapes task influenced thought probe responses and later cueing effects was explored. Panel 1 displays recall accuracy as a function of Age Group, Experiment, and cue-probe proximity in the Shapes task (closer than the median distance between cues and probes vs. farther than the median distance between cues and probes). Panel 2 displays standardized response latencies as a function of Age Group, Experiment, and cue-probe proximity in the Shapes task. 